TRANSACTIONS OF THE

AMERICAN MATHEMATICAL SOCIETY

Volume 353, Number 9, Pages 3803-3831

S 0002-9947(01)02801-X

Article electronically published on April 24, 2001

\title{
THE SERRE SPECTRAL SEQUENCE OF A MULTIPLICATIVE FIBRATION
}

\author{
YVES FÉLIX, STEPHEN HALPERIN, AND JEAN-CLAUDE THOMAS
}

\begin{abstract}
In a fibration $\Omega F \stackrel{\Omega j}{\rightarrow} \Omega X \stackrel{\Omega \pi}{\rightarrow} \Omega B$ we show that finiteness conditions on $F$ force the homology Serre spectral sequence with $\mathbb{F}_{p}$-coefficients to collapse at some finite term. This in particular implies that as graded vector spaces, $H_{*}(\Omega X)$ is "almost" isomorphic to $H_{*}(\Omega B) \otimes H_{*}(\Omega F)$. One consequence is the conclusion that $X$ is elliptic if and only if $B$ and $F$ are.
\end{abstract}

\section{INTRODUCTION}

In this paper we consider the fibration

$$
\Omega F \stackrel{\Omega j}{\longrightarrow} \Omega X \stackrel{\Omega \pi}{\longrightarrow} \Omega B
$$

obtained by looping a fibration of simply connected pointed spaces of finite type. Working over a prime field $\mathbb{F}_{p}, p>0$, we analyse the relationship of the loop space homologies $H_{*}(\Omega F), H_{*}(\Omega X)$ and $H_{*}(\Omega B)$ in the presence of a finiteness condition on $F$, and show that at this level the loop space $\Omega X$ behaves much like the product $\Omega F \times \Omega B$.

The starting point for this and the other results in this paper is the structure of $H_{*}(\Omega F)$ as a cocommutative Hopf algebra, with the Alexander-Whitney diagonal and multiplication induced from composition of loops. The category of cocommutative Hopf algebras, which includes the universal enveloping algebras of graded Lie algebras, was studied by Milnor and Moore 27. and Moore and Smith 29], who defined such notions as 'Hopf center', 'Hopf kernel of a morphism' and 'normal sub and quotient Hopf algebras', generalizing the classical versions for graded Lie algebras. If $H$ is a normal sub Hopf algebra of $G$, the Hopf quotient is denoted by $G / / H$.

The study of the relationship of $H_{*}(\Omega X), H_{*}(\Omega F)$ and $H_{*}(\Omega B)$ in the fibration $\left.{ }^{*}\right)$ was begun by Browder in [6], via the Serre spectral sequence $\left(E^{r}, d^{r}\right)$. He showed that this was a spectral sequence of cocommutative Hopf algebras, and then used a sophisticated application of his biprimitive spectral sequence to obtain precise information about the differentials in the Serre spectral sequence; this process, however, "loses" the structure of $E^{r}$ as a Hopf algebra.

Received by the editors January 30, 1998.

2000 Mathematics Subject Classification. Primary 57T25, 55R20, 57T05.

Key words and phrases. Multiplicative fibration, loop space, Hopf algebra, Serre spectral sequences, elliptic space.

Research of the second author was partially supported by an NSERC operating grant. Research of the first and third authors was partially supported by UMR-6093 au CNRS.

This work was also partially supported by a NATO travel grant held by all three authors.

(C)2001 American Mathematical Society 
A different approach to the homology of loop space fibrations is taken by Moore and Smith ([29] and [30]), who rely on the Eilenberg-Moore spectral sequence. This is particularly effective for principal fibrations with fibre $K(\pi, n)$, since they pullback from fibrations with base $K(\pi, n+1)$ whose cohomology is a free commutative graded algebra, which simplifies the computation of the $E^{2}$-term.

In this paper we focus on fibrations $(*)$ for which $F$ satisfies a finiteness condition (e.g. $F$ is finite dimensional, or $F$ has finite LS category). This is in the context of the special properties such a condition is already known to impose on the Hopf algebra $H_{*}(\Omega F)$. For example suppose $F$ is an $r$-connected $n$-dimensional $\mathrm{CW}$ complex. Then:

- If $k \geq n / r$ then $\alpha^{p^{k}}=0, \alpha \in H^{+}(\Omega F)$ (Proposition 4.12, below).

- If $p \geq n /(r-1)$ then $H_{*}(\Omega F)$ is a universal enveloping algebra [20].

Moreover, if $F$ has finite LS category, then

- The Betti numbers dim $H_{k}(\Omega F)$ grow either polynomially or semi-exponentially in $k$, with the latter case being the generic one [15.

For our purpose here, the key result $[8]$ is that for any simply connected $F$ :

$$
\begin{aligned}
\max \left\{n \mid H_{n}(F) \neq 0\right\} & \geq \operatorname{cat}(F) \\
& \geq \operatorname{depth} H_{*}(\Omega F) \\
& \geq \operatorname{depth} \text { Hopf center of } H_{*}(\Omega F),
\end{aligned}
$$

where the depth of an augmented algebra $A$ is the least $m$ (or $\infty$ ) such that $\operatorname{Ext}_{A}^{m}\left(\mathbb{F}_{p}, A\right) \neq 0$, and cat $(-)$ is the Lusternik-Schnirelmann category of a space or map. Now commutative cocommutative Hopf algebras of finite depth are finitely generated as algebras [10. Thus the finiteness of any of the invariants above implies that the Hopf center of $H_{*}(\Omega F)$ is a finitely generated algebra. Consequently in this paper we will focus on fibrations $\left(^{*}\right)$ satisfying that condition; i.e. we will assume that

$$
\text { The Hopf centre of } H_{*}(\Omega F) \text { is a finitely generated algebra. }
$$

(Unfortunately, even when $F$ is a finite complex and with rational coefficients there is no known general formula for an upper bound on the number of generators of the Hopf center of $H_{*}(\Omega F)$.)

The main topological results of the paper are summarized in Theorem A-D below, which follow from Theorems 4.2, 4.7, 3.9 and Theorem 5.6.

Theorem A. If (**) holds then there are isomorphisms of graded vector spaces,

$$
H_{*}(\Omega X) \otimes A \cong H_{*}(\Omega F) \otimes H_{*}(\Omega B) \otimes \Lambda V
$$

in which $A$ is the finite tensor product of monogenic Hopf algebras and $\Lambda V$ is the exterior algebra on a finite dimensional vector space concentrated in odd degrees.

Remark. If $F$ and $B$ have finite LS category then generically $H(\Omega F)$ and $H(\Omega B)$ will grow at least semi-exponentially, as observed above. Thus in this case Theorem A shows that $H_{*}(\Omega X), H_{*}(\Omega F)$ and $H_{*}(\Omega B)$ are "large" compared with $A$ and $\Lambda V$, and so $H(\Omega X)$ and $H(\Omega B) \otimes H(\Omega F)$ are "approximately linearly isomorphic".

A monogenic Hopf algebra is either an exterior algebra $\Lambda x$ on an element $x$ of odd degree, or a polynomial algebra $\mathbb{F}_{p}[y]$ on an element $y$ of even degree, or a 
truncated polynomial algebra of the form $\mathbb{F}_{p}[y] / y^{p^{t}}$, again with deg $y$ even. Thus in Theorem A the Hilbert series $A(z)=\sum_{0}^{\infty}\left(\operatorname{dim} A_{n}\right) z^{n}$ has the form

$$
A(z)=\prod_{i=1}^{q}\left(1+z^{2 n_{i}+1}\right) \prod_{j=1}^{r}\left(1-z^{2 m_{j}}\right)^{-1} \prod_{k=1}^{s} \frac{\left(1-z^{2 p t_{k} l_{k}}\right)}{\left(1-z^{2 l_{k}}\right)} .
$$

In particular, $r$ is just the rate of polynomial growth of $A(z)$, poly $\operatorname{gr} A(z)$. Moreover, since $A(z)=\prod_{i=1}^{q}\left(1+z^{2 n_{i}+1}\right) g\left(z^{2}\right)$ it follows that $q$ is also an invariant of $A(z)$; we call it the index, ind $A(z)$. In Proposition 4.15 we show that

$$
\operatorname{dim} V=\operatorname{ind} A(z)-\text { poly } \operatorname{gr} A(z) .
$$

Next we have

Theorem B. Suppose the Hopf centre of $H_{*}(\Omega F)$ is generated by $k$ elements. Then :

(i) At most $2 k$ differentials in the Serre spectral sequence are non-zero. In particular it collapses at some $E^{r}, r<\infty$.

(ii) The Hopf kernel of $H_{*}(\Omega j)$ is a finite tensor product of monogenic Hopf algebras, and the Hopf quotient, Hopf ker $H_{*}(\Omega \pi) / / \operatorname{Im} H_{*}(\Omega j)$ is an exterior algebra on finitely many primitive elements of odd degree.

Theorem B contains the core assertion of this paper: that condition $(* *)$ forces the collapse of the spectral sequence at some finite stage. For the convenience of the reader we outline the main ingredients of the proof (the section by section description at the end of the introduction indicates the location of each step):

- The Hopf kernel of $H_{*}(\Omega j), K(\Omega j)$, is central in $H_{*}(\Omega F)$. (This follows from the existence of a natural adjoint representation of $H_{*}(\Omega X)$ in $H_{*}(\Omega F)$.) Hence if the Hopf center of $H_{*}(\Omega F)$ is generated by $k$ elements, so is $K(\Omega j)$. - In the homology Serre spectral sequence $\left(E^{r}, d^{r}\right)$ for the fibration $\left(^{*}\right)$, let $K^{r}$ be the Hopf kernel of $E_{0, *}^{2} \rightarrow E_{0, *}^{r}$ and let $Z^{s}$ be the Hopf kernel of $E_{0, *}^{s} \rightarrow E_{0, *}^{s+1}$. Then $K(\Omega j)=\bigcup_{r} K^{r}$ and $K^{r}=\bigotimes_{s=2}^{r-1} Z^{s}$. (The second assertion follows from a "splitting lemma" which can be applied to the short exact sequence of Hopf algebras $K^{s-1} \rightarrow K^{s} \rightarrow Z^{s-1}$ because we are able to limit the possible degrees of generators for each $Z^{s}$.) In particular, if $\lambda(-)$ denotes the minimal number of generators of a connected graded algebra then

$$
\lambda\left(\text { Hopf center } H_{*}(\Omega F)\right) \geq \lambda\left(K(\Omega j)=\sum_{s=2}^{\infty} \lambda\left(Z^{s}\right)\right) .
$$

- The non-triviality of the differential $d^{r}$ forces the existence of a generator in some $Z^{s}$, some $s \leq r$. A generator can be "used" at most once in this way, and so

$$
\#\left\{r \mid d^{r} \neq 0\right\} \leq \sum_{s=2}^{\infty} \lambda\left(Z^{s}\right) .
$$

(This argument depends on a careful analysis of the structure of the Hopf algebra $E^{r}$ and the form of the differential $d^{r}$.) 
We also have

Theorem C. (i) If cat $j<\infty$, then the Serre spectral sequence for $\left(^{*}\right)$ collapses at some $E^{r}, r<\infty$, if and only if $H_{*}(\Omega F)$ has finite depth.

(ii) If $H_{*}(F)$ is finite dimensional, then depth $H_{*}(\Omega F) \leq$ cat $\pi$.

Theorems $\mathrm{A}-\mathrm{C}$ are illustrated by the fibration

$$
\xi: F \stackrel{j}{\rightarrow} S^{2 p} \cup_{p} e^{2 p+1} \stackrel{f}{\rightarrow} S^{3},
$$

where $f_{\mid S^{2 p}}$ represents a non-trivial element of $\pi_{2 p}\left(S^{3}\right)$. In Example 3.6 we show that the Serre spectral sequence for $\Omega \xi$ collapses at $E^{3}$. Since

$$
\text { cat } j \leq \operatorname{cat}\left(S^{2 p} \cup_{p} e^{2 p+1}\right)=1
$$

it follows from Theorem $\mathrm{C}$ that $H_{*}(\Omega F)$ has finite depth and thus that condition $\left({ }^{* *}\right)$ holds. Here we have (subscripts denote degrees) $H_{*}\left(\Omega S^{3}\right)=T\left(a_{2}\right)$ and $H_{*}\left(\Omega\left(S^{2 p} \cup_{p} e^{2 p+1}\right)\right)=T\left(y_{2 p-1}, b_{2 p}\right)$, while the calculations of the example imply that

$$
H_{*}(\Omega F)=\Lambda x_{1} \otimes T(y,[b, y],[b,[b, y]], \cdots),
$$

and that in Theorem A we may take

$$
A=\Lambda x_{1} \otimes \mathbb{F}_{p}[a] / a^{p} \text { and } \Lambda V=\Lambda y .
$$

As a second example, take $p=3$ and let $F$ be the homogeneous space $F_{4} / \operatorname{Spin}(9)$, 28], and consider the fibration

$$
\Omega F \stackrel{\Omega j}{\longrightarrow} \operatorname{Spin}(9) \stackrel{\Omega \pi}{\longrightarrow} F_{4}
$$

obtained by looping $F \stackrel{j}{\rightarrow} B \operatorname{Spin}(9) \stackrel{\pi}{\rightarrow} B F_{4}$. The homology of $\operatorname{Spin}(9)$ and $F_{4}$ are given, respectively, by $\Lambda\left(x_{3}, x_{7}, x_{11}, x_{15}\right)$ and by $\Lambda\left(x_{3}, x_{7}, x_{11}, x_{15}\right) \otimes \mathbb{F}_{3}\left[x_{8}\right] / x_{8}^{3}$, where subscripts denote degrees [28]. In particular, the Serre spectral sequence for the fibration $\operatorname{Spin}(9) \rightarrow F_{4} \rightarrow F$ collapses at the $E^{2}$-term, which implies that $H^{*}(F)=\mathbb{F}_{3}\left[x_{8}\right] / x_{8}^{3}$.

It also follows that $H_{*}(\Omega \pi)$ is injective, with image $\Lambda\left(x_{3}, x_{7}, x_{11}, x_{15}\right)$ and that $H_{+}(\Omega j)=0$. A straightforward Serre spectral sequence calculation for the fibration $\Omega F \rightarrow \operatorname{Spin}(9) \rightarrow F_{4}$ then gives $H_{*}(\Omega F)=\Lambda y_{1} \otimes \mathbb{F}_{3}\left[z_{22}\right]$. Thus in Theorem A we may take

$$
A=\Lambda y_{1} \otimes \mathbb{F}_{3}\left[z_{22}\right] \otimes \mathbb{F}_{3}\left[x_{8}\right] / x_{8}^{3} \quad \text { and } \quad V=0 .
$$

The methods in this paper depend on the coefficient field having chararcteristic $p>0$. However the results remain true over $\mathbb{Q}$ and, in fact, were established much earlier in [19] and 7] using Sullivan's minimal models. Moreover, with rational coefficients Theorem A has the form

$$
H_{*}(\Omega X ; \mathbb{Q}) \otimes \Lambda s W \otimes \mathbb{Q}[W] \cong H_{*}(\Omega F ; \mathbb{Q}) \otimes H_{*}(\Omega B ; \mathbb{Q}),
$$

with $W$ a finite dimensional vector space concentrated in even degrees and the suspension $s W$ defined by $(s W)_{k}=W_{k-1}$. The examples above make it clear that this stronger version fails in positive characteristic.

Among the examples and applications we would like to draw attention to Theorem 5.5, which deals with elliptic spaces. This is a remarkable class of 1-connected finite complexes $Y$, introduced in [11, and characterized by the fact that for each prime $q$ the loop space homology $H_{*}\left(\Omega Y ; \mathbb{F}_{q}\right)$ grows polynomially. Here we establish a result announced in [11]: 
Theorem D. If $F, X$ and $B$ are finite 1-connected complexes, then $X$ is elliptic if and only if $F$ and $B$ are.

We have divided the paper into two parts, putting the topology first and the algebra second, so that the second part is almost self-contained and the first part depends on the results it contains. Our justification is that the main results are in the first part, the second part is much more technically complex, and the quotations from it in the first part while essential, are relatively few in number. The organization of the material is sketched in the following outline.

\section{PART I: LOOP SPACE FIBRATIONS}

$\S 1$. Graded Hopf algebras. This recalls the standard definitions and constructions in this category, such as the Hopf kernel of a morphism and the Hopf quotient $H / / K$. It also has the "splitting lemma" (Proposition 1.2) referred to in the sketch above of the proof of Theorem B. This is a slight strengthening of the standard lemma used to prove the Borel structure theorem: every connected commutative graded Hopf algebra is isomorphic as an algebra to a tensor product of monogenic Hopf algebras.

$\S 2$. Adjoint action of a loop space. Here is constructed the homotopy adjoint action of $\Omega X$ on $\Omega F$, analogous to the classical adjoint action of a group on a normal subgroup. This in turn determines a natural adjoint representation of $H_{*}(\Omega X)$ in $H_{*}(\Omega F)$ compatible with the classical adjoint representation of the graded Hopf algebras in themselves, and defined even trough $H_{*}(\Omega j): H_{*}(\Omega F) \rightarrow H_{*}(\Omega X)$ may not be injective.

$\S 3$. Homology of a loop space fibration. Here we introduce the Hopf kernels, images and Hopf cokernels of the morphisms $H_{*}(\Omega F) \stackrel{H_{*}(\Omega j)}{\longrightarrow} H_{*}(\Omega X) \stackrel{H_{*}(\Omega \pi)}{\longrightarrow}$ $H_{*}(\Omega B)$ associated with the fibration $(*)$. They are denoted respectively by $K(-)$, $\operatorname{Im}(-)$ and $C(-)$. The first key observation (Proposition 3.3) is that $K(\Omega j)$ is central in $H_{*}(\Omega F)$, which follows from the properties of the adjoint representation constructed in $\S 2$.

The section is completed with two main results. Proposition 3.8 states that $\rho_{k}(\Omega X) \leq \rho_{k}(\Omega B)+\rho_{k}(\Omega F)$, where $\rho_{k}(-)$ is the sum of the dimensions of the primitive subspaces in odd degrees $\leq k$. Theorem 3.9 establishes the relations between the depths of the Hopf algebras $K(\Omega j), H_{*}(\Omega F)$ and $H_{*}(\Omega B)$ and the LS category of the maps $j$ and $\pi$. These imply Theorem C.

$\S 4$. Collapse of the Serre spectral sequence. This contains the main topological results of the paper. Theorem 4.2 is a more precise version of Theorem B, while Theorem 4.7 asserts that the collapse at a finite stage of the Serre spectral sequence for $\left(^{*}\right)$ is equivalent to the finite generation of either $K(\Omega j)$ or of the commutative algebra $C(\Omega \pi)^{\vee}$ dual to the coalgebra $C(\Omega \pi)$. Theorem 4.7 is then used to prove Theorem A. Both these results depend heavily on the analysis of the Hopf algebra structure of the spectral sequence carried out in $\S 6$ and $\S 7$.

Finally, Proposition 4.12 states that if $B$ is $n$-dimensional and $r$-connected then all $p^{k}$-powers vanish in $H^{+}(\Omega B), k \geq n / r$. This is used in Theorem 4.7 to show that if $B$ is finite dimensional and if the Serre spectral sequence for $(*)$ collapses then $C(\Omega \pi)$ is finite dimensional. 
§5. Examples and applications. Aside from the examples this contains an analogue (Theorem 5.5) of the Borel transgression theorem: the case when $K(\Omega j)$ is a polynomial algebra. Here $C(\Omega \pi)$ must be an exterior coalgebra, with primitive elements transgressing to the generators of $K(\Omega j)$, and the vector space $V$ of Theorem A is zero. This section also contains the proof of Theorem D (Theorem 5.6).

\section{Part II: Differential Hopf algebras}

§6. Bigraded differential Hopf algebras. The principal assertion about the Serre spectral sequence for the fibration $\left(^{*}\right)$ is that each term has the form

$$
E^{r} \cong E_{*, 0}^{r} \otimes \Lambda U^{r} \otimes E_{0, *}^{r} \quad \text { (as Hopf algebras), }
$$

where $\Lambda U^{r}$ is the exterior Hopf algebra on a finite dimensional space $U^{r}$ of primitive elements concentrated in odd degrees. (This is a refinement of Browder's result in 6].) We work abstractly with bigraded differential Hopf algebras of this form, and with certain restrictions on the bidegrees of $U^{r}$, and show that $H\left(E^{r}\right)$ then has the same form.

Indeed if $Z=K\left(E_{0, *}^{r} \rightarrow E_{0, *}^{r+1}\right)$ and if $W=C\left(E_{*, 0}^{r+1} \rightarrow E_{*, 0}^{r}\right)$, then it follows from 13 that

$$
H\left(E^{r}\right) \cong H_{*, 0}\left(E^{r}\right) \otimes H\left(W \otimes \Lambda U^{r} \otimes Z\right) \otimes H_{0, *}\left(E^{r}\right) .
$$

Moreover, it turns out that $W$ and $Z$ are bicommutative Hopf algebras, and so we can bypass Browder's biprimitive spectral sequence to show directly that $H\left(W \otimes \Lambda U^{r} \otimes \Lambda Z\right)$ has the form $\Lambda V$.

$\S 7$. Spectral sequences of Hopf algebras. The main result here is the explicit Hopf algebra structure of a spectral sequence $\left(E^{r}, d^{r}\right)$ of cocommutative bigraded Hopf algebras beginning with $E^{2}=E_{*, 0}^{2} \otimes E_{0, *}^{2}$. This is provided in Theorem 7.2, and is an immediate consequence of the results in $\S 6$. Also of importance is Theorem 7.3, which asserts that if the Hopf kernel $K$ of $E_{0, *}^{2} \rightarrow E_{0, *}^{\infty}$ is commutative, then there is an algebra isomorphism $K \cong \bigotimes_{s \geq 2} Z^{s}$, where $Z^{s}$ is the Hopf kernel of $E_{0, *}^{s} \rightarrow E_{0, *}^{s+1}$. As noted above this plays a key role in the proof of Theorem B.

\section{PART I: LOOP SPACE FIBRATIONS}

\section{Graded Hopf algebras}

We work over a field $\mathbb{K}$ and recall that a graded vector space $V$ is a family $\left\{V_{i}\right\}_{i \in \mathbb{Z}}$ of vector spaces; we also write this as $\left\{V^{j}\right\}_{j \in \mathbb{Z}}$ by setting $V_{i}=V^{-i}$. The dual of $V$ is the graded vector space $V^{\vee}$ defined by $V_{i}^{\vee}=\operatorname{Hom}\left(V_{-i}, \mathbb{K}\right)$.

A graded coalgebra over a field $\mathbb{K}$ is a graded vector space $C$ equipped with an associative comultiplication $\Delta: C \rightarrow C \otimes C$ and a counit $\varepsilon: C \rightarrow \mathbb{K}$; if $C$ is further equipped with a coalgebra morphism $\mathbb{K} \rightarrow C$ then the primitive subspace $P(C)$ consists of the elements $x \in C$ satisfying $\Delta x=x \otimes 1+1 \otimes x$.

A graded algebra $A$ is a graded vector space with associative multiplication and unit $\mathbb{K} \rightarrow A ; A$ is connected if $A=\mathbb{K} \oplus A_{+}$or if $A=\mathbb{K} \oplus A^{+}$. In this case the space $Q A$ of indecomposables of $A$ is defined by $Q A=A_{+} /\left(A_{+} \cdot A_{+}\right)$or by $Q A=A^{+} /\left(A^{+} \cdot A^{+}\right)$.

A graded Hopf algebra is a graded vector space $H$ that is simultaneously a graded algebra with unit $\mathbb{K} \rightarrow H$ and a graded coalgebra, and such that the comultiplication and counit are algebra morphisms. If $H$ is connected, then the primitive and 
indecomposable spaces are both defined and the quotient map to $Q H$ restricts to the important linear map

$$
P H \longrightarrow Q H \text {. }
$$

If $H$ is a connected graded Hopf algebra, then $([27 ; 8.4)$ there is a unique antiautomorphism $\omega$ of $H$ such that mult $\circ(\omega \otimes i d) \circ \Delta$ is the counit $H \rightarrow \mathbb{K}$. This is called the conjugation in $H$ and satisfies $\omega^{2}=i d$ if $H$ is cocommutative. The adjoint representation of a commutative connected graded Hopf algebra in itself is defined by $\operatorname{Ad} x(y)=\sum_{i}(-1)^{\operatorname{deg} y \operatorname{deg} x_{i}^{\prime \prime}} x_{i}^{\prime} y \omega\left(x_{i}^{\prime \prime}\right)$, where $\Delta x=\sum x_{i}^{\prime} \otimes x_{i}^{\prime \prime}[33]$, [10]; $\S 2)$.

If a graded Hopf algebra, $H$, is represented in graded vector spaces $V$ and $W$ then the diagonal representation in $V \otimes W$ is that given by $H \stackrel{\Delta}{\longrightarrow} H \otimes H \longrightarrow$ $\operatorname{End}(V) \otimes \operatorname{End}(W)$. A representation of $H$ in a graded coalgebra $C$ (resp. in a graded algebra $A$, or in a graded Hopf algebra, $G$ ) is a representation in which the comultiplication (resp., the multiplication, both structure maps) are morphisms of $H$-modules. In the case of coalgebras this is equivalent to requiring that the action $H \otimes C \rightarrow C$ be a morphism of coalgebras. In particular, as observed in ([10]; $(2.2)$ and (2.3) (ii)) the adjoint representation of a graded cocommutative Hopf algebra is a representation of a Hopf algebra in a Hopf algebra.

Remark. If a Hopf algebra is represented in an algebra, then the primitive subspace acts by derivations.

Let $H$ be a connected graded Hopf algebra. If $K$ is a sub Hopf algebra then $H / H K_{+}$(or $H / H K^{+}$) inherits the structure of a graded coalgebra, denoted by $H / / K$ and, as shown in [27], there is an isomorphism $K \otimes H / / K \cong H$ of $K$ modules. A sub Hopf algebra $K$ is normal if $K_{+} H=H K_{+}\left(\right.$or $\left.K^{+} H=H K^{+}\right)$, in which case the quotient map induces a graded Hopf algebra structure in $H / / K$. This is the quotient Hopf algebra. If $\varphi: H \rightarrow G$ is a morphism of cocommutative connected graded Hopf algebras, then the Hopf kernel of $\varphi$ is the normal sub Hopf algebra $K \subset H$ given by $K=\{x \in H \mid(\varphi \otimes i d) \Delta x=1 \otimes x\}$. The Hopf kernel of $H \rightarrow H / / K$ is $K$. Finally, the Hopf center of $H$ is the union of the central sub Hopf algebras of $H$.

Suppose $H=\mathbb{K} \oplus H^{>0}$ is a commutative graded Hopf algebra. The Borel structure theorem ([4]; Theorem 3.2) asserts that $H$ is isomorphic as a graded algebra with the tensor product of monogenic Hopf algebras. We shall need a somewhat stronger version of this theorem. Fortunately the proof given in ([27; Proposition 7.10) can be slightly modified to give us the result we need. We include this for the convenience of the reader.

Proposition 1.2. Suppose $A \longrightarrow B \stackrel{\pi}{\longrightarrow} C$ is a short exact sequence of connected, commutative (not necessarily cocommutative) Hopf algebras such that the spaces of indecomposables satisfy: for pn even and $k \geq 1$,

$$
Q_{n}(C) \neq 0 \Rightarrow Q_{n p^{k}}(A)=0 .
$$

Assume $\mathbb{K}$ is a perfect field. Then $\pi$ admits a splitting $\sigma: C \rightarrow B$ of graded algebras; moreover, if $B$ is primitively generated $\sigma$ can be taken to be a splitting of Hopf algebras.

Proof. Reduce in the standard way to the case that $C=\mathbb{K}[y] / y^{p^{l}}$ with $p \operatorname{deg} y$ even and that each $A_{i}$ is finite dimensional. Denote by $\xi: x \rightarrow x^{p}$ the Frobenius 
morphism and consider the short exact sequences of Hopf algebras,

$$
A / / \xi^{l}(A) \rightarrow B / / \xi^{l}(A) \rightarrow C .
$$

To simplify notation put $A^{\prime}=A / / \xi^{l}(A)$.

Now choose $z \in B / / \xi^{l}(A)$ to represent $y$. The reduced diagonal of $z$ is in $A_{+}^{\prime} \otimes A_{+}^{\prime}$ for trivial degree reasons, and so $z^{p^{l}}$ is primitive. Since $z^{p^{l}}$ maps to zero in $C$, it is even a primitive element of $A^{\prime}: z^{p^{l}} \in A^{\prime}$.

On the other hand, clearly $Q(A)$ maps onto $Q\left(A^{\prime}\right)$, and so $Q\left(A^{\prime}\right)$ vanishes in degrees of the form $p^{k} \operatorname{deg} z, k \geq 1$. Thus $Q\left(\xi^{i-1} A^{\prime}\right)$ vanishes in degrees of the form $p^{k} \operatorname{deg} z, k \geq i$. Hence if $s=p^{l} \operatorname{deg} z$ then $Q_{s}\left(\xi^{i-1} A^{\prime}\right)=0, i \leq l$. Thus from the exact sequences

$$
0 \rightarrow P\left(\xi A^{\prime}\right) \rightarrow P\left(\xi^{i-1} A^{\prime}\right) \rightarrow Q\left(\xi^{i-1} A^{\prime}\right)
$$

([27; Proposition 4.21) we deduce that $P_{s}\left(\xi^{l} A^{\prime}\right) \stackrel{\cong}{\longrightarrow} P_{s}\left(A^{\prime}\right)$. In particular, $z^{p^{l}} \in$ $\xi^{l}\left(A_{+}^{\prime}\right)=0$.

Let $\omega \in B$ represent $z$. Then the reduced diagonal of $\omega$ is in $A_{+} \otimes A_{+}$. Since $z^{p^{l}}=0, \omega^{p^{l}} \in \xi^{l}\left(A_{+}\right) \cdot B$, and hence $\omega^{p^{l}} \in \xi^{l}\left(A_{+}\right)$. Write $\omega^{p^{l}}=a^{p^{l}}, a \in A_{+}$, and define $\sigma$ by $\sigma(y)=\omega-a$.

Finally, if $B$ is primitively generated so is $A$. Thus we may choose $z$ and $\omega$ above to be primitive. Then $\omega^{p^{l}}$ is a primitive element in $\xi^{l}(A)$ and hence the $p^{l}$-th power of a primitive element in $A$; i.e. we may take $a \in A_{+}$above to be primitive as well. In this case $\sigma$ is a splitting of Hopf algebras.

Proposition 1.2. implies the following result of Lin ([22]; Lemma 2.2.2).

Corollary $1.3(22])$. If $i$ is odd or if $p$ is odd and $i$ is not divisible by $2 p$, then the functor $P_{i}$ is exact when applied to short exact sequences of cocommutative connected graded Hopf algebras.

Corollary 1.4. If $H$ is a connected cocommutative graded Hopf algebra, then the maps

$$
P_{i}(G) \longrightarrow Q_{i}(G)
$$

are surjective if $i$ is odd or if $p$ is odd and $i$ is not divisible by $2 p$.

\section{Adjoint ACtion OF A LOOP SPACE}

Throughout this section we consider a fixed Hurewicz fibration of path connected spaces,

$$
\xi: F \stackrel{j}{\rightarrow} X \stackrel{\pi}{\rightarrow} B,
$$

in which $B$ is also 1 -connected. It loops to the multiplicative fibration

$$
\Omega F \stackrel{\Omega j}{\longrightarrow} \Omega X \stackrel{\Omega \pi}{\longrightarrow} \Omega B,
$$

where here and elsewhere $\Omega$ denotes the Moore loop space.

Now recall [36], [16], 12] that any continuous map $f: U \longrightarrow V$ can be naturally converted to a fibration, whose fibre, the homotopy fibre of $f$, is equipped with a natural left action of $\Omega V$. (Note that some authors, e.g. [16], [12] use the equivalent right action). In the case of the fibration, (2.1), the fibre $F$ is naturally homotopy equivalent to the homotopy fibre of $\pi$, and this defines a "homotopy action" of $\Omega B$ on $F$. We will often abuse language and refer simply to the action of $\Omega B$ on $F$. 
We review a number of examples that will be important for the sequel. First, note that the homotopy action, $\mu: \Omega B \times F \longrightarrow F$, loops to the homotopy action for $(2.2)$,

$$
\Omega \mu: \Omega^{2} B \times \Omega F \longrightarrow \Omega F
$$

as follows directly from the definitions. Next, consider the free loop space fibration

$$
\Omega X \rightarrow X^{S^{1}} \stackrel{\rho}{\rightarrow} X
$$

with $\rho(f)=f(*)$, where $*$ denotes the base point of $S^{1}$. The corresponding action of $\Omega X$ on itself is given by (cf. [24]; Prop. 3.2)

$$
A d: \Omega X \times \Omega X \longrightarrow \Omega X, \quad A d(f)(g)=f g f^{-1}
$$

we call this the adjoint action of $\Omega X$ on itself. Note that Milnor [26] shows that $\Omega X$ is equivalent to a topological group, and this equivalence identifies the adjoint action with conjugation.

We generalize this adjoint action in the context of fibration (2.1), by constructing a natural "adjoint" action $A d_{\xi}$ of $\Omega X$ on $\Omega F$, such that the diagram

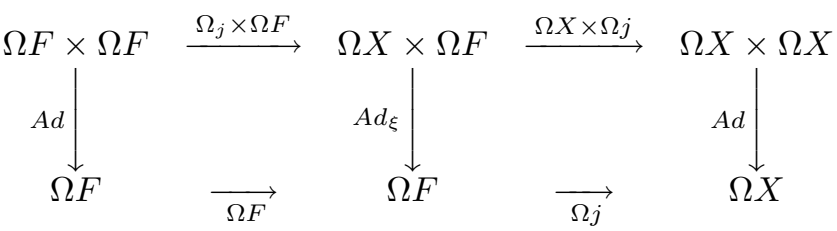

homotopy commutes. This could be done (cf. [18]; Prop. 3.1.1) via Milnor's method, by converting (2.2) to a short exact sequence of topological groups $K \longrightarrow$ $G \longrightarrow G / K$ and using conjugation by $G$ in $K$. We shall instead give a more elementary description.

Let $c_{z}$ denote the constant loop at $z \in B$ and consider the pull-back diagram

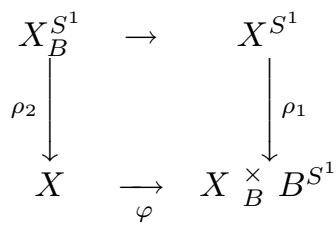

in which: $\rho_{1}(f)=(f(*), \pi \circ f), \varphi(x)=\left(x, c_{\pi x}\right)$ and $X_{B}^{S^{1}}$ is the subspace of $X^{S^{1}}$ of those maps $f: S^{1} \longrightarrow X$ such that $\pi f$ is constant; i.e., whose image is contained in some fibre of $\pi$. An easy exercise exhibits $\rho_{1}$ as a fibration, with fibre $\Omega F$. Hence $\rho_{2}$ is also a fibration with fibre $\Omega F$.

Definition 2.7. The homotopy action of $\Omega X$ on $\Omega F$ determined by the pullback fibration $\rho_{2}$ is called the adjoint action corresponding to the fibration $\xi$, and is denoted by $A d_{\xi}: \Omega X \times \Omega F \longrightarrow \Omega F$.

Remark 2.8. Applying Definition 2.7 to the fibration $X \stackrel{i d_{X}}{\longrightarrow} X \longrightarrow *$ we recover the classical adjoint action described above.

Lemma 2.9. With the hypotheses and notation above:

(i) The adjoint action of $\Omega X$ on $\Omega F$ is natural (up to homotopy) in $\xi$.

(ii) The diagram (2.5) homotopy commutes. 
(iii) The multiplication $\Omega F \times \Omega F \longrightarrow \Omega F$ is equivariant up to homotopy with respect to the adjoint action of $\Omega X$ in $\Omega F$ and the induced diagonal action of $\Omega X$ in $\Omega F \times \Omega F$.

Proof. (i) is true by construction. To see (ii) simply apply (i) to the maps of fibrations

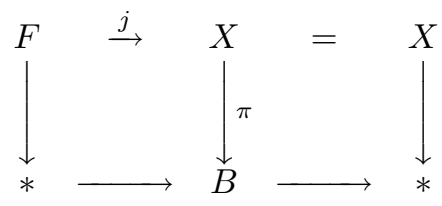

For (iii) apply the naturality of the homotopy action in the fibre to the map of fibrations

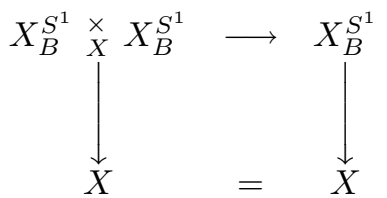

defined by composition of loops.

Next, note that the fibration $\Omega^{2} B \longrightarrow B^{S^{2}} \longrightarrow B$ defines an action of $\Omega B$ on $\Omega^{2} B$. Thus $\Omega X$ acts on $\Omega^{2} B$ via this action and $\Omega \pi$. As in the proof of Lemma 2.9, naturality arguments using diagram (2.6) yield

Lemma 2.10. With the hypotheses and notation above, the map $\Omega \mu: \Omega^{2} B \times$ $\Omega F \longrightarrow \Omega F$ is equivariant up to homotopy with respect to the actions of $\Omega X$.

In passing from geometry to algebra, we work over a fixed ground field $\mathbb{K}$ and write $H_{*}(-)$ for $H_{*}(-; \mathbb{K})$. Thus if $X$ is a pointed space, multiplication in $\Omega X$ makes $H_{*}(\Omega X)$ into a cocommutative graded Hopf algebra. The self-map $f \mapsto f^{-1}$ of $\Omega X$ induces an antiautomorphism $\omega$ such that mult $\circ(\omega \otimes i d) \circ \Delta$ is the augmentation $H_{*}(\Omega X) \longrightarrow \mathbb{K}$. Thus if $X$ is 1 -connected, then $\omega$ coincides with the conjugation in $H_{*}(\Omega X)$.

Analogously, an action of $\Omega X$ on a space $Y$ defines an action of the Hopf algebra $H_{*}(\Omega X)$ on the coalgebra $H_{*}(Y)$. In particular the adjoint action of $\Omega X$ in itself defines the representation

$$
H_{*}(A d): H_{*}(\Omega X) \otimes H_{*}(\Omega X) \longrightarrow H_{*}(\Omega X),
$$

of $H_{*}(\Omega X)$ in itself, and it is immediate from the definition that this is the adjoint representation of $H_{*}(\Omega X)$.

Definition 2.11. The adjoint representation of $H_{*}(\Omega X)$ in $H_{*}(\Omega F)$ corresponding to the fibration $\xi: F \longrightarrow X \longrightarrow B$ is the representation induced by the adjoint action of $\Omega X$ in $\Omega F$. It will be denoted by $x \otimes y \mapsto A d_{\xi}(x)(y), x \in H_{*}(\Omega X)$, $y \in H_{*}(\Omega F)$.

Lemma 2.9 translates to:

Lemma 2.12. With the hypotheses and notation above, let $x \in H_{*}(\Omega X)$, and $y, z \in H_{*}(\Omega F)$. Then 
(i) $A d_{\xi}$ is a representation of the Hopf algebra $H_{*}(\Omega X)$ in the Hopf algebra $H_{*}(\Omega F)$.

(ii) $(A d y) z=A d_{\xi}\left(H_{*}(\Omega j) y\right) z$;

(iii) $\operatorname{Adx}\left(H_{*}(\Omega j) y\right)=H_{*}(\Omega j)\left(A d_{\xi}(x) y\right)$.

\section{Homology of A LOOP SPACE FiBRATion}

We work over a fixed prime field $\mathbb{F}_{p}$ of characteristic $p>0$ and write $H_{*}(-)=$ $H_{*}\left(-; \mathbb{F}_{p}\right)$.

Fix a fibration

$$
\Omega \xi: \Omega F \stackrel{\Omega j}{\longrightarrow} \Omega X \stackrel{\Omega \pi}{\longrightarrow} \Omega B
$$

obtained by looping a fibration of simply connected pointed spaces, such that for each $i$ and $j, H_{i}(B)$ and $H_{j}(F)$ are finite dimensional. Thus each $H_{i}(\Omega B)$ and $H_{j}(\Omega F)$ are also finite dimensional.

With the fibration $\Omega \xi$ is associated the standard homology Serre spectral sequence $\left(E^{r}, d^{r}\right), r \geq 2$. By $([6] ; \S 5)$ this is a spectral sequence of bigraded Hopf algebras, in which the $E^{\infty}$-term is the bigraded Hopf algebra associated with the canonical filtration of $H_{*}(\Omega X)$ and the $E^{2}$-term is the Hopf algebra $H_{*}(\Omega B) \otimes H_{*}(\Omega F)$. In particular the results of $\S 7$ apply verbatim.

The structure of $H_{*}(\Omega X)$ itself is best understood in the context of the Hopf algebra morphisms:

$$
H_{*}(\Omega F) \stackrel{H_{*}(\Omega j)}{\longrightarrow} H_{*}(\Omega X) \stackrel{H_{*}(\Omega \pi)}{\longrightarrow} H_{*}(\Omega B) .
$$

We therefore introduce the notation:

$$
\begin{aligned}
K(\Omega j) & =\operatorname{Hopf} \operatorname{ker} H_{*}(\Omega j) ; \\
\operatorname{Im}(\Omega j) & =\operatorname{Im} H_{*}(\Omega j) ; \\
K(\Omega \pi) & =\operatorname{Hopf} \operatorname{ker} H_{*}(\Omega \pi) ; \\
\operatorname{Im}(\Omega \pi) & =\operatorname{Im} H_{*}(\Omega \pi) ; \\
C(\Omega \pi) & =H_{*}(\Omega B) / / \operatorname{Im} H_{*}(\Omega \pi) .
\end{aligned}
$$

Note that $C(\Omega \pi)=H_{*}(\Omega B) \otimes_{\operatorname{Im}(\Omega \pi)} \mathbb{F}_{p}$ is in general not a Hopf algebra, but only a coalgebra $(\S 1)$ and an $H_{*}(\Omega B)$-module. Finally for any path connected space $Z$ we let $P(Z)$ be the primitive subspace:

$$
P(Z)=P\left(H_{*}(Z)\right)
$$

Proposition 3.3. In the fibration (3.1):

(i) $K(\Omega j)$ is central in $H_{*}(\Omega F)$.

(ii) [18] $\operatorname{Im}(\Omega j)$ is normal in $H_{*}(\Omega X)$.

(iii) [30] $\operatorname{Im}(\Omega j) \subset K(\Omega \pi)$, and the quotient $K(\Omega \pi) / / \operatorname{Im}(\Omega j)$ is the exterior algebra $\Lambda V$ on the space $V$ of primitive elements of odd degree.

Proof. (i) Lemma 2.12(ii) implies that if $y, z \in H_{*}(\Omega F)$ and if $H_{*}(\Omega j) y=0$, then $(\operatorname{Ad} y)(z)=0$. This holds in particular for all $y \in K_{+}(\Omega j)$, and so $K(\Omega j)$ is central, by ([10]; Lemma 2.3(iii)). 
(ii) It follows from Lemma 2.12(iii) that $\operatorname{Im}(\Omega j)$ is invariant under the operators $\operatorname{Ad} x, x \in H_{*}(\Omega X)$. Thus this sub Hopf algebra $\operatorname{Im}(\Omega j)$ is normal, by ([10]; Lemma 2.4(i)).

(iii) It follows from ([6]; Theorem 5.8), (see also Theorem 7.2, below) that

$$
E_{*, *}^{\infty}=E_{*, 0}^{\infty} \otimes \Lambda U_{*, *}^{\infty} \otimes E_{0, *}^{\infty},
$$

where $U^{\infty}$ is concentrated in odd degrees. Since $K(\Omega \pi) / / \operatorname{Im}(\Omega j) \cong \Lambda U^{\infty}$ as graded vector spaces we have only to apply Proposition 1.2 and Corollary 1.4. Different proofs are given for (iii) in [30] and ([18]; Prop. 3.1.1).

Corollary 3.4. With the hypotheses and notation above:

(i) There is a graded subspace $W \subset P_{\text {odd }}(\Omega X)$, isomorphic with $V$, and an isomorphism of filtered graded coalgebras

$$
f: \Lambda W \otimes \operatorname{Im}(\Omega j) \stackrel{\cong}{\cong} K(\Omega \pi),
$$

extending the identity in $\operatorname{Im}(\Omega j)$. (W is given the filtration induced from that of $H_{*}(\Omega X)$.)

(ii) If $p>2$ there is an isomorphism of graded coalgebras,

$$
H_{*}(\Omega X) / / \operatorname{Im}(\Omega j) \cong \operatorname{Im}(\Omega \pi) \otimes \Lambda V .
$$

Proof. (i) By Corollary 1.3 there is a subspace $W \subset P_{\text {odd }}(K(\Omega \pi))$ that maps isomorphically to $V$ under the surjection $K(\Omega \pi) \longrightarrow \Lambda V$ of Proposition 3.3(iii). Let $w_{i}$ be a basis of $W$ compatible with the filtration and define

$$
f\left(w_{i_{1}} \wedge \cdots \wedge w_{i_{k}} \otimes z\right)=w_{i_{1}} \cdots \cdots w_{i_{k}} \cdot z, \quad z \in \operatorname{Im}(\Omega j), \quad i_{1}<\cdots<i_{k} .
$$

(ii) The dual of the inclusion $\Lambda V \longrightarrow H_{*}(\Omega X) / / \operatorname{Im}(\Omega j)$ has a multiplicative splitting because $(\Lambda V)^{\vee}$ is an exterior algebra generated in odd degrees, and $p>2$.

Remark 3.5. Since the elements $w_{i} \in W$ (in the proof of 3.4(i)) are primitive, they act by derivations $\theta_{i}$ in $\operatorname{Im}(\Omega j)$. The elements $c_{i}=w_{i}^{2}$ and $b_{i j}=\left[w_{i}, w_{j}\right]$ are primitive and of even degree; in view of the isomorphism $f$ we have $c_{i}, b_{i j} \in \operatorname{Im}(\Omega j)$. The multiplication in $\Lambda W \otimes \operatorname{Im}(\Omega j)$ induced by $f$ is determined in an obvious way by the multiplication in $\operatorname{Im}(\Omega j)$, the $\theta_{i}$ and the elements $c_{i}, b_{i j}$.

Example 3.6. We give a simple example in which the $\theta_{i}$ and $c_{i}$ are non-zero. All spaces are to be considered as localized at an odd prime $p$. Let $S^{3}\langle 3\rangle$ denote the 3 -connected cover of $S^{3}$, so that we have the fibre sequence

$$
\mathbb{C} P^{\infty} \rightarrow S^{3}\langle 3\rangle \stackrel{\lambda}{\rightarrow} S^{3} \rightarrow K\left(\mathbb{Z}_{(p)}, 3\right) .
$$

Loop this sequence.

Then $H_{*}\left(\Omega S^{3}\right)=T\left(a_{2}\right), T(-)$ denoting the tensor algebra, and $a_{2}^{p}$ vanishes in $H\left(K\left(\mathbb{Z}_{(p)}, 2\right)\right)$. Thus $a_{2}^{p}$ is a primitive element in $K(\Omega \pi)$. By Corollary 3.4(i) it is in the image of $H\left(\Omega S^{3}\langle 3\rangle\right)$. On the other hand the first step in constructing $K\left(\mathbb{Z}_{(p)}, 3\right)$ from $S^{3}$ is to cone a map $f: S^{2 p} \cup_{p} e^{2 p+1} \longrightarrow S^{3}$. Thus $f$ factors through $\lambda$ to identify $S^{2 p} \cup_{p} e^{2 p+1}$ as the $(2 p+1)$-skeleton of $S^{3}\langle 3\rangle$. Hence $a_{2}^{p}$ is in the image of $H_{*}(\Omega f)$. But $H_{*}\left(\Omega\left(S^{2 p} \cup_{p} e^{2 p+1}\right)\right)=T\left(y_{2 p-1}, b_{2 p}\right)$, subscripts denoting degrees. It follows that $H_{*}(\Omega f) y_{2 p-1}=0$ and $H_{*}(\Omega f) b_{2 p}=a_{2}^{p}$. Hence

$$
K(\Omega f)=T(y,[b, y],[b,[b, y]], \ldots) .
$$


Let $F$ be the homotopy fibre of $f$ and consider the multiplicative fibration

$$
\Omega \xi: \Omega F \longrightarrow \Omega\left(S^{2 p} \cup e^{2 p+1}\right) \stackrel{\Omega f}{\longrightarrow} \Omega S^{3} .
$$

Since $S^{2 p} \cup_{p} e^{2 p+1} \longrightarrow S\langle 3\rangle$ is $(2 p+1)$-connected, looping the induced map of fibres gives a $2 p$-connected map $\Omega F \longrightarrow \Omega \mathbb{C} P^{\infty}=S^{1}$. Thus $H_{2 p-1}(\Omega F)=0$.

Now consider the Serre spectral sequence for $\Omega \xi$. Since $E^{\infty}$ begins in degree $2 p-1$, we have $E^{2}=\mathbb{F}_{p}\left[a_{2}\right] \otimes E_{0, *}^{2}$ with $0 \neq d^{2} a_{2}=x \in E_{0,1}^{2}$. Thus $E^{3}=$ $\mathbb{F}_{p}\left[a_{2}^{p}\right] \otimes \Lambda\left(a_{2}^{p-1} x\right) \otimes E_{0, *}^{3}$. Since $H_{2 p-1}(\Omega F)=0, a_{2}^{p-1} x$ must represent the class $y_{2 p-1}$, while we already know that $a_{2}^{p}$ represents $b_{2 p}$. These classes therefore survive to $E^{\infty}$, which implies $E^{3}=E^{\infty}$. Thus in the Hopf kernel $T(y,[b, y],[b,[b, y]], \cdots)$ of $H_{*}(\Omega f)$, the element $y$ corresponds to a primitive element in $E_{+,+}^{\infty} \cdot$ Its square $y^{2}$, and its adjoint action in $\operatorname{Im} H(\Omega j)$ are clearly non-zero.

The fibration $\xi: F \rightarrow X \rightarrow B$ induces a principal fibration $\eta: \Omega X \stackrel{\Omega \pi}{\rightarrow} \Omega B \rightarrow F$.

Proposition 3.7. If $H_{*}(\Omega B) \rightarrow H_{*}(F)$ is surjective, then $H_{*}(\Omega F)$ is commutative and $H^{*}(F) \cong C(\Omega \pi)^{\vee}$ as graded algebras.

Proof. Our hypothesis implies that the Serre spectral sequence for $\eta$ collapses at $E^{2}$, and so $H(\Omega \pi)$ is injective. This implies that $\operatorname{Im}(\Omega j)=\mathbb{F}_{p}$ so that $H_{*}(\Omega F)=$ $K(\Omega j)$ is abelian. Also, from the collapse at $E^{2}$ for $\eta$, we deduce that $C(\Omega \pi)=$ $H_{*}(\Omega B) \otimes_{\operatorname{Im}(\Omega \pi)} \mathbb{F}_{p} \cong H_{*}(F)$ as coalgebras and as modules over $H_{*}(\Omega B)$.

Proposition 3.8. In the fibration (3.1), the primitive subspaces of $H_{*}(\Omega X)$, $H_{*}(\Omega B)$ and $H_{*}(\Omega F)$ satisfy

$$
\sum_{i \leq k} \operatorname{dim} P_{2 i+1}(\Omega X) \leq \sum_{i \leq k}\left(\operatorname{dim} P_{2 i+1}(\Omega B)+\operatorname{dim} P_{2 i+1}(\Omega F)\right), k \geq 0 .
$$

Proof. We apply Theorem 7.2 to the Serre spectral sequence for $\Omega \xi$, writing $E^{r}=$ $E_{*, 0}^{r} \otimes \Lambda U^{r} \otimes E_{0, *}^{r}, r \geq 2$, with $U^{r}$ concentrated in odd degrees and $U^{\infty}=\underset{r}{\lim } U^{r}$. The remarks preceding Proposition 6.6 identify $\Lambda U^{r+1}$ as $H\left(W \otimes \Lambda U^{r} \otimes Z\right)$, where in the sequence

$$
E_{*, 0}^{r+1} \stackrel{\sigma}{\rightarrow} E^{r} \stackrel{\kappa}{\rightarrow} E_{0, *}^{r+1}
$$

Hopf ker $\kappa / / I m \sigma=W \otimes \Lambda U^{r} \otimes Z$. Since (Corollary 1.3) $P_{\text {odd }}$ is exact, we have

$$
P_{\text {odd }}^{r} \cong P_{\text {odd }, 0}^{r+1} \oplus P_{\text {odd }}\left(W \otimes \Lambda U^{r} \otimes Z\right) \oplus P_{0, \text { odd }}^{r+1}
$$

and

$$
P_{\text {odd }}^{r+1} \cong P_{\text {odd }, 0}^{r+1} \oplus U^{r+1} \oplus P_{0, \text { odd }}^{r+1} .
$$

As is evident from Corollary 6.9, the creation of basis elements of $U^{r+1}$ in addition to those surviving from $U^{r}$ is at the cost of eliminating primitive basis elements of lower odd degree from $W \otimes \Lambda U^{r} \otimes Z$. Hence

$$
\sum_{i \leq k} \operatorname{dim} P_{2 i+1}\left(E^{r+1}\right) \leq \sum_{i \leq k} \operatorname{dim} P_{2 i+1}\left(E^{r}\right) .
$$

Use induction to replace $r+1$ by $\infty$ and $r$ by 2 in this inequality, and then use Corollary 1.3 to get $P_{\text {odd }}\left(E^{\infty}\right) \cong P_{\text {odd }}(\Omega X)$. 
Recall from the introduction that cat(-) denotes the LS category of spaces and maps and that for a connected graded algebra $A$, depth $A$ is the least $m$ (or $\infty$ ) such that $\operatorname{Ext}_{A}^{m}\left(\mathbb{F}_{p}, A\right) \neq 0$. Analogously, the grade of an $A$-module $M, \operatorname{gr}_{A}(M)$, is the least $m$ (or $\infty)$ such that $\operatorname{Ext}_{A}^{m}(M, A) \neq 0$.

Theorem 3.9. In the fibration (3.1):

(i) depth $K(\Omega j) \leq \operatorname{depth} H_{*}(\Omega F) \leq$ cat $j+\operatorname{depth} K(\Omega j)$.

(ii) If $H_{*}(F)$ is finite dimensional then depth $H_{*}(\Omega B) \leq$ cat $\pi$.

Proof. (i) The first inequality follows via the Hochschild-Serre spectral sequence 9] from the fact that $K(\Omega j)$ is a normal sub Hopf algebra of $H_{*}(\Omega F)$. To prove the second, we may clearly assume $K(\Omega j)$ has finite depth. Thus, since $K(\Omega j)$ is commutative, from the exact sequence $K(\Omega j) \longrightarrow H_{*}(\Omega F) \longrightarrow \operatorname{Im}(\Omega j)$ of Hopf algebras we deduce ([8]; Proposition 3.1) that

$$
\operatorname{depth} H_{*}(\Omega F)=\operatorname{depth} K(\Omega j)+\operatorname{depth} \operatorname{Im}(\Omega j) .
$$

Next, consider the short exact sequence

$$
\operatorname{Im} \Omega j \longrightarrow K(\Omega \pi) \longrightarrow \Lambda V
$$

of Hopf algebras (Proposition 3.3). Again, because $K(\Omega j)$ is commutative and has finite depth, it is finitely generated [10]. Hence (Proposition 7.5) the Serre spectral sequence collapses at some finite $r$. This implies (Theorem $7.2(\mathrm{v})$ ) that $\Lambda V$ is a finite dimensional exterior algebra. In the Hochschild-Serre spectral sequence [9] corresponding to the Hopf algebra exact sequence $\operatorname{Im}(\Omega j) \rightarrow K(\Omega j) \rightarrow \Lambda V$ :

$$
\operatorname{Ext}_{\Lambda V}^{s}\left(\mathbb{F}_{p}, \operatorname{Ext} t_{I m(\Omega j)}^{t}\left(\mathbb{F}_{p}, K(\Omega \pi)\right)\right) \Rightarrow \operatorname{Ext}_{K(\Omega \pi)}^{s+t}\left(\mathbb{F}_{p}, K(\Omega \pi)\right) .
$$

We may therefore identify $\operatorname{Ext}_{\operatorname{Im}(\Omega j)}\left(\mathbb{F}_{p}, K(\Omega \pi)\right)$ as the free $\Lambda V$-module on $\operatorname{Ext}_{I m(\Omega j)}\left(\mathbb{F}_{p}, \operatorname{Im} \Omega j\right)$, as follows from ([8]; Lemma 3.5). Since $\operatorname{Ext}_{\Lambda V}^{*}\left(\mathbb{F}_{p}, \Lambda V\right)=$ $\operatorname{Ext}_{\Lambda V}^{0}\left(\mathbb{F}_{p}, \Lambda V\right)$ this implies that

$$
\operatorname{depth} \operatorname{Im}(\Omega j)=\operatorname{depth} K(\Omega \pi) .
$$

Put $G=H_{*}(\Omega X), K=K(\Omega \pi)$ and $H=H_{*}(\Omega B)$. Because $G$ is a free $K$-module we may identify

$$
\operatorname{Ext}_{G}(G / / K, G)=\operatorname{Ext}_{K}\left(\mathbb{F}_{p}, G\right)=\prod_{\alpha} \operatorname{Ext}_{K}\left(\mathbb{F}_{p}, K\right) \cdot g_{\alpha},
$$

where $g_{\alpha}$ represents a basis for $G / / K$. On the other hand $G / / K=\operatorname{Im}(\Omega \pi)$ and the $G$-module $H=H_{*}(\Omega B)$ is thus a direct sum of copies of $\operatorname{Im}(\Omega \pi)$. Hence

$$
\operatorname{Ext}_{G}(H, G) \cong \prod_{\beta} \operatorname{Ext}_{G}(G / / K, G) \cdot h_{\beta} .
$$

These two equations show that

$$
\operatorname{grade}_{H_{*}(\Omega X)}\left(H_{*}(\Omega B)\right)=\operatorname{depth} K(\Omega \pi) .
$$

Now, consider the fibration $\Omega B \rightarrow F \stackrel{j}{\rightarrow} X$. The induced action of $H_{*}(\Omega X)$ on $H_{*}(\Omega B)$ is via multiplication and the homomorphism $H_{*}(\Omega \pi)$, and so by (14]; Theorem A and (1.2))

$$
\operatorname{grade}_{H_{*}(\Omega X)}\left(H_{*}(\Omega B)\right) \leq \operatorname{cat} j .
$$

Combining (3.10), (3.11), (3.12) and (3.13) we achieve the second inequality of (i). 
(ii) As in part (i) we may apply (14]; Theorem A and (1.2)) to deduce

$$
\operatorname{grade}_{H_{*}(\Omega B)}\left(H_{*}(F)\right) \leq \operatorname{cat} \pi \text {. }
$$

If $H_{*}(F)$ is finite dimensional a trivial argument shows that $\operatorname{grade}_{H_{*}(\Omega B)}\left(H_{*}(F)\right)=$ $\operatorname{depth} H_{*}(\Omega B)$.

\section{Collapse of the Serre spectral sequence}

We continue to work over $\mathbb{F}_{p}, p>0$, and to write $H(-)=H\left(-; \mathbb{F}_{p}\right)$.

As in $\S 3$ we fix a fibration

$$
\Omega \xi: \Omega F \stackrel{\Omega j}{\longrightarrow} \Omega X \stackrel{\Omega \pi}{\longrightarrow} \Omega B
$$

obtained by looping a fibration of simply connected pointed spaces in which each $H_{i}(B)$ and $H_{j}(F)$ is finite dimensional. The homology Serre spectral sequence of $\Omega \xi$ will be denoted by $\left(E^{r}, d^{r}\right), r \geq 2$. We continue to use the notation (3.2), and for any topological space $Y$ we denote by $Y(z)$ the Hilbert series $H(Y)(z)=$ $\sum\left[\operatorname{dim} H_{i}(Y)\right] z^{i}$.

Let $P^{r}$ denote the primitive subspace of the $r$ th term $E^{r}$. Since (Corollary 1.4) every indecomposable element of odd degree in a cocommutative Hopf algebra is represented by a primitive element, it follows that $d^{r}=0$ if and only if

$$
d^{r}\left(P_{\text {odd }}^{r}\right)=0=\operatorname{Im} d^{r} \cap P_{\text {odd }}^{r} .
$$

Recall $(\S 1)$ that $Q A=A_{+} /\left(A_{+} \cdot A_{+}\right)$is the space of indecomposables of a connected graded algebra $A$, so that $\operatorname{dim} Q A$ is the minimal number of generators of $A$.

Theorem 4.2. Suppose in the fibration (4.1) that the Hopf centre of $H_{*}(\Omega F)$ is generated as an algebra by $k$ elements. Then

$$
\sum_{s=2}^{\infty}\left(\operatorname{dim} d^{s}\left(P_{o d d}^{s}\right)+\operatorname{dim}\left(\operatorname{Im} d^{s} \cap P_{o d d}^{s}\right)\right) \leq 2 \operatorname{dim} Q K(\Omega j) \leq 2 k .
$$

In particular, at most $2 k$ of the differentials in the spectral sequence are non-zero and the spectral sequence collapses at some $E^{r}, r<\infty$.

For the proof of the theorem we need to recall the standard (and easy)

Lemma 4.3. If $H \subset G$ is an inclusion of commutative (not necessary cocommutative) connected Hopf algebras, then $\operatorname{dim} Q H \leq \operatorname{dim} Q G$.

Proof of Theorem 4.2. Let $K^{r}$ be the Hopf kernel of the edge homomorphism $E_{0, *}^{2}$ $\longrightarrow E_{0, *}^{r}$. We shall show that for each $r \geq 2$,

$$
\sum_{s=2}^{r}\left(\operatorname{dim} d^{s}\left(P_{o d d}^{s}\right)+\operatorname{dim}\left(\operatorname{Im} d^{s} \cap P_{o d d}^{s}\right)\right) \leq 2 \operatorname{dim} Q K^{r} .
$$

Now, by Theorem 3.3(i), $K^{r} \subset K(\Omega j) \subset$ Hopf centre of $H_{*}(\Omega F)$. Use Lemma 4.3 to replace $2 \operatorname{dim} Q K^{r}$ by $2 \operatorname{dim} Q K(\Omega j)$ in (4.4).

In proving (4.4) we rely on $\S 6$ and $\S 7$ and use the notation established there without further reference. We may, of course, assume that $\operatorname{dim} Q K^{r}=k_{r}<\infty$. Moreover, Theorem 7.3 applies and gives

$$
K^{r} \cong \bigotimes_{s=2}^{r-1} Z^{s} \quad \text { (as graded algebras) }
$$

with $Z^{s}$ the Hopf kernel of $E_{0, *}^{s} \longrightarrow E_{0, *}^{s+1}$. 
Now consider the structure of $Z^{s}$ as analyzed in Proposition 6.6. Its generators are in bijection with a basis of $d^{s}\left(E_{s, 0}^{s}\right) \oplus d^{s}\left(U_{s, *}^{s}\right)$ where, as always in $\S 7$, we write $E^{s}=E_{*, 0}^{s} \otimes \Lambda U^{s} \otimes E_{0, *}^{s}$. Moreover $d^{s}$ vanishes on the decomposable elements in $E_{s, 0}^{s}$. Since every indecomposable element of odd degree is represented by a primitive, and since the derivative of an odd primitive is an even primitive, and hence in $E_{0, *}^{s}$, the number $\ell_{s}$ of generators of $Z^{s}$ of even degree satisfies

$$
\ell_{s} \geq \operatorname{dim} d^{s}\left(P_{o d d}^{s}\right) .
$$

Next observe that $I m d^{s} \cap P_{o d d}^{s}$ consists of $d^{s}\left(E_{s, 0}^{s}\right)$ (when $s$ is even) together with $U_{*, s-1}^{s} \cap d^{s}\left(E_{*, 0}^{s}\right)$. The elements in $U_{*, s-1}^{s}$ have bidegrees $(i, s-1)$ with $i<s-1$; cf. Theorem 7.2(iv). Thus in view of the analysis in $\S 6$, (cf. Corollary 6.9), they were created at an earlier stage by the destruction by some $d^{q}$ of a generator of $Z^{q}$ of finite height. Moreover when $s$ is even the number, $m_{s}$, of generators of $Z^{s}$ of odd degree satisfies

$$
m_{s} \geq \operatorname{dim} d^{s}\left(E_{s, 0}^{s}\right)
$$

In this way we see that

$$
\sum_{s \leq r} m_{s}+\sum_{s \leq r} l_{s} \geq \sum_{s \leq r} \operatorname{dim}\left(\operatorname{Im} d^{s} \cap P_{o d d}^{s}\right) .
$$

This, and the inequality (4.5) give (4.7), and the theorem.

Theorem 4.2 shows that the finite generation of $K(\Omega j)$ forces the collapse of the Serre spectral sequence of (4.1). We shall now see that in turn the collapse of the spectral sequence imposes, and is often equivalent to, finiteness restrictions on the other Hopf algebras (or coalgebras) in (3.2).

To describe these conditions we introduce one more piece of notation: $\Lambda x$ will denote the exterior algebra on an element $x$, and $\mathbb{F}_{p}(x)$ will denote a monogenic Hopf algebra; thus $\mathbb{F}_{p}(x)=\Lambda x, \mathbb{F}_{p}[x]$ or $\mathbb{F}_{p}[x] / x^{p^{k}}$. More generally we shall denote by $\mathbb{F}_{p}\left(x_{1}, \ldots, x_{t}\right)$ a commutative graded algebra generated by elements $x_{1}, \ldots, x_{t}$ and built inductively out of subalgebras $\mathbb{F}_{p}\left(x_{1}, \ldots, x_{i}\right)$ by conditions of the form:

$$
\mathbb{F}_{p}\left(x_{1}, \ldots, x_{i+1}\right)= \begin{cases} & \mathbb{F}_{p}\left(x_{1}, \ldots, x_{i}\right) \otimes \mathbb{F}_{p}\left(x_{i+1}\right) \\ \text { or } & \\ & \mathbb{F}_{p}\left(x_{1}, \ldots, x_{i}\right) \otimes \mathbb{F}_{p}\left[x_{i+1}\right] / f .\end{cases}
$$

In the second case we require $f=x_{i+1}^{p^{k}}+\sum_{j<p^{k}} a_{j} x_{i+1}^{j}$ and $p \operatorname{deg} x_{i+1}$ is even.

Theorem 4.7. (i) If the Serre spectral sequence for the fibration (4.1) collapses at some $E^{r}, r<\infty$, then the commutative graded algebras

$$
K(\Omega j), \quad K(\Omega \pi) / / \operatorname{Im} \Omega j \quad \text { and } \quad C(\Omega \pi)^{\vee}
$$

are all finitely generated.

(ii) Conversely, if either $K(\Omega j)$ or $C(\Omega \pi)^{\vee}$ are finitely generated then the Serre spectral sequence collapses at some $E^{r}, r<\infty$. In this case there are isomorphisms of graded algebras:

$$
K(\Omega j) \cong \mathbb{F}_{p}\left(x_{1}\right) \otimes \cdots \otimes \mathbb{F}_{p}\left(x_{s}\right), \quad C(\Omega \pi)^{\vee} \cong \mathbb{F}_{p}\left(y_{1}, \ldots, y_{t}\right)
$$

and

$$
K(\Omega \pi) / / I m \Omega j \cong \Lambda\left(v_{1}\right) \otimes \cdots \otimes \Lambda\left(v_{q}\right)
$$


(iii) If the spectral sequence collapses at some $E^{r}, r<\infty$, and if $H_{*}(B)$ is finite dimensional then

$$
\operatorname{dim} C(\Omega \pi)<\infty
$$

Corollary 4.8. If the Serre spectral sequence of $\xi$ collapses at some $E^{r}$, then for any pull-back $\xi^{\prime}$ of $\xi$ the spectral sequence for $\Omega \xi^{\prime}$ also collapses, although possibly at a later stage.

Proof of 4.8. It suffices to note that $K\left(\Omega j^{\prime}\right) \subset K(\Omega j)$. The theorem asserts that $K(\Omega j)$ is finitely generated. Hence so is $K\left(\Omega j^{\prime}\right)$ (Lemma 4.3 ) and we apply the theorem again.

Remark. There are examples of non-multiplicative fibrations with spectral sequences collapsing at some $E^{r}, r \geq 3$, but admitting pull-backs whose spectral sequences never collapse.

Corollary 4.9. If cat $j<\infty$ then the spectral sequence collapses at some $E^{r}$, $r<\infty$, if and only if $H_{*}(\Omega F)$ has finite depth. In this case

$$
\text { depth } K(\Omega \pi)=\text { depth } \operatorname{Im}(\Omega j) \leq \text { cat } j
$$

and

$$
\text { depth } H_{*}(\Omega F)=\text { depth } K(\Omega j)+\text { depth } \operatorname{Im}(\Omega j) .
$$

Proof of 4.9. If $H_{*}(\Omega F)$ has finite depth then the collapse is asserted in Theorem 4.2. Conversely if the spectral sequence collapses the theorem asserts that $K(\Omega j)$ is finitely generated. It is also commutative (Proposition 3.3), and hence has finite depth [10], 9]. Moreover ([10]; Proposition 3.1) gives

$$
\text { depth } H_{*}(\Omega F)=\operatorname{depth} K(\Omega j)+\operatorname{depth} \operatorname{Im}(\Omega j) \text {. }
$$

Now we may apply Theorem 3.9 to conclude that depth $\operatorname{Im}(\Omega j) \leq$ cat $j$. Since $K(\Omega \pi) / / \operatorname{Im}(\Omega j)$ is finite dimensional it follows (see $[9]$ ) that depth $K(\Omega \pi)=$ depth $\operatorname{Im}(\Omega j)$.

Corollary 4.10. If $H_{*}(\Omega j)=0$ and the spectral sequence collapses at some $E^{r}$, $r<\infty$, then (cf. §2) $H_{*}(\Omega F)$ is finitely generated and commutative and $K(\Omega \pi)$ is a finite dimensional exterior algebra.

Corollary 4.11. If the spectral sequence collapses at some $E^{r}, r<\infty$, then for some $N$ the sequence of primitive spaces,

$$
0 \longrightarrow P_{n}(\Omega F) \longrightarrow P_{n}(\Omega X) \longrightarrow P_{n}(\Omega B) \longrightarrow 0
$$

is exact for all $n \geq N$ such that either $n$ is odd, or $p$ is odd and $n$ is not divisible by $2 p$.

Proof. This is immediate from the theorem and Corollary 1.3.

Proof of Theorem 4.7. Proposition 7.5 asserts that equivalence of collapse with either of the conditions: $K(\Omega j)$ (resp., $\left.(\Omega \pi)^{\vee}\right)$ is a finitely generated algebra.

Now suppose the spectral sequence collapses at $E^{r}, r<\infty$. Since $K(\Omega j)$ is a finitely generated commutative Hopf algebra it is, as a graded algebra, the finite tensor product of monogenic Hopf algebras. Moreover, we have the finite sequence of inclusions

$$
\operatorname{Im}(\Omega \pi)=E_{*, 0}^{r} \hookrightarrow E_{*, 0}^{r-1} \hookrightarrow \cdots \hookrightarrow E_{*, 0}^{2}=H_{*}(\Omega B)
$$


Since (Theorem 7.2(ii)) the inclusions $E_{*, 0}^{i+1} \hookrightarrow E_{*, 0}^{i}$ are normal and the dual commutative Hopf algebras $\left(E_{*, 0}^{i} / / E_{*, 0}^{i+1}\right)^{\vee}$ are finitely generated it follows that $C(\Omega \pi)^{\vee}$ has the form $\mathbb{F}_{p}\left(y_{1}, \ldots, y_{t}\right)$. Observe as well (Proposition 3.3(iii) and its proof) that $K(\Omega \pi) / / \operatorname{Im} \Omega j=\Lambda V$, with $V \cong U^{\infty}=U^{r}$. Thus $V$ is finite dimensional ([6]; Theorem 5.8 or Theorem $7.2(\mathrm{v}))$.

Finally, if $H_{*}(B)$ is finite dimensional as well, then by Proposition 4.12, below, for some fixed $k$, all $\left(p^{k}\right)$-th powers vanish in $H^{+}(\Omega B)$. Since this property passes to subquotients the finitely generated algebras $\left(E_{*, 0}^{i} / / E_{*, 0}^{i+1}\right)^{\vee}$ are finite dimensional, and so $C(\Omega \pi)$ itself is finite dimensional too.

Proposition 4.12. Suppose the reduced homology $H_{*}(X)$ of a simply connected space is concentrated in degrees $i \in[r+1, n]$ and let $k$ be the least integer such that $p^{k} \geq n / r$. Then

(i) All $p^{k}$-th powers vanish in $H^{+}(\Omega X)$.

(ii) There is a finite sequence

$$
\mathbb{F}_{p}=B(0) \subset B(1) \subset \cdots \subset B(k)=H_{*}(\Omega X)
$$

of normal sub Hopf algebras of $H_{*}(\Omega X)$ such that each $B(i+1) / / B(i)$ is primitively generated.

Remark 4.13. If $p \geq n / r$ and $X$ is finite then this is a theorem of Anick ([1]; Theorem 9.1) in response to a conjecture of McGibbon and Wilkerson (25]; p.699). It is related to a result of Lannes and Schwartz (21]; Proposition 0.6), which asserts that the Steenrod algebra acts on $H^{*}(\Omega X)$ with finite dimensional orbits.

Proof of Proposition 4.12. Using the fact (27]; Proposition 4.20) that a connected cocommutative graded Hopf algebra $H$ is primitively generated if and only if the $p$ th powers vanish in $H^{\vee}$, it is easy to see that conditions (i) and (ii) are equivalent. We prove (i).

Consider the cohomology Serre spectral sequence for the fibration $\Omega X \longrightarrow$ $P X \longrightarrow X$. Suppose $p$ is odd. If $\alpha \in H^{2 m}(\Omega X)$ survives until the term $E_{q+1}^{0,2 m}$ and if $\alpha^{p} \neq 0$, then $\alpha^{p}=\mathcal{P}^{m}(\alpha)$ survives until $E_{q p+1}^{0,2 m p}$ by a theorem of Araki [2] and Vasquez [35] - cf. also ([24]; Theorem 6.10). Thus if $\alpha^{p^{k}} \neq 0$, it survives until $E_{q p^{k}+1}^{*, *}$. Now $H(X)$ is $r$-connected and so all of $E_{2}^{0, *}$ survives until $E_{r+1}^{0, *}$. Hence for any $\alpha$ of even degree in $H^{+}(\Omega X)$, if $\alpha^{p^{k}} \neq 0$ it survives until $E_{r p^{k}+1}^{* *}$. But since $H^{>n}(X)=0, E_{n+1}^{*, *}=E_{\infty}^{*, *}=\mathbb{F}_{p}$. It follows that $\alpha^{p^{k}}=0$ if $r p^{k} \geq n$. The case $p=2$ is proved in the same way.

Next, we show how to deduce Theorem A of the introduction from Theorem 4.7. Indeed, (cf. §1) we have isomorphisms of graded vector spaces

$$
H(\Omega B) \cong \operatorname{Im}(\Omega \pi) \otimes C(\Omega \pi), \quad H(\Omega F) \cong K(\Omega j) \otimes \operatorname{Im}(\Omega j)
$$

and

$$
H(\Omega X) \cong K(\Omega \pi) \otimes \operatorname{Im}(\Omega \pi) \cong \operatorname{Im}(\Omega j) \otimes[K(\Omega \pi) / / \operatorname{Im}(\Omega j)] \otimes \operatorname{Im}(\Omega \pi) .
$$

Since $K(\Omega \pi) / / \operatorname{Im}(\Omega j) \cong \Lambda V$ these give the relation

$$
H(\Omega X) \otimes C(\Omega \pi) \otimes K(\Omega j) \cong H(\Omega B) \otimes H(\Omega F) \otimes \Lambda V .
$$

Under the hypotheses of Theorem A, $E^{r}=E^{\infty}$, some $r<\infty$. Thus, as observed in Theorem 4.7, $V$ is finite dimensional and $C(\Omega \pi)^{\vee}$ and $K(\Omega j)$ are finitely generated 
commutative graded Hopf algebras. Thus the latter two are both isomorphic as algebras to a finite tensor product of monogenic Hopf algebras (4]; Theorem 3.2), and this proves Theorem A.

Finally, recall the definitions of index and polynomial growth in the introduction. Set $\chi=$ poly gr - index.

Proposition 4.15. If the Serre spectral sequence for the fibration (4.1) collapses at some $E^{r}, r<\infty$, then

$$
\operatorname{dim} V=-\chi(K(\Omega j))-\chi(C(\Omega \pi)) .
$$

Proof. Put $K(i)=\operatorname{Hopf} \operatorname{ker}\left(E_{0, *}^{i} \rightarrow E_{0, *}^{2}\right), C(i)=E_{*, 0}^{2} / / E_{*, 0}^{i}$ and, as in $\S 7, E^{i}=$ $E_{*, 0}^{i} \otimes \Lambda U^{i} \otimes E_{*, 0}^{i}$. We show by induction that

$$
\operatorname{dim} U^{i}=-\chi\left(K^{i}\right)-\chi\left(C^{i}\right), \quad 2 \leq i \leq r,
$$

which is the desired conclusion when $i=r$. Now both sides of (4.16) are obviously zero for $i=2$. For the inductive step, note that $K^{i+1} \cong K^{i} \otimes Z^{i}$ and $\left(C^{i+1}\right)^{\vee} \cong\left(C^{i}\right)^{\vee} \otimes A_{i}$ as graded spaces; here $Z^{i}=\operatorname{Hopf} \operatorname{ker}\left(E_{0, *}^{i} \rightarrow E_{0, *}^{i+1}\right)$ and $A_{i}=\left(E_{*, 0}^{i+1} / / E_{*, 0}^{i}\right)^{\vee}$. Now Corollary 6.9 gives an explicit description of how $U^{i+1}$ is constructed from $U^{i}$, depending on the multiplicative structures in $Z^{i}$ and $A_{i}$. From this it follows at once that

$$
\operatorname{dim} U^{i+1}-\operatorname{dim} U^{i} \equiv-\chi\left(Z^{i}\right)-\chi\left(A_{i}\right) .
$$

This, together with the trivial observation that $\chi(M \otimes N)=\chi(M)+\chi(N)$, gives the inductive step.

\section{EXAMPLES AND APPLICATIONS}

We continue to work over $\mathbb{F}_{p}$, with the notation and conventions of $\S 4$. In particular

$$
\Omega \xi: \Omega F \stackrel{\Omega j}{\longrightarrow} \Omega X \stackrel{\Omega \pi}{\longrightarrow} \Omega B
$$

is a fibration obtained by looping a fibration $\xi$ of simply connected pointed spaces in which each $H_{i}(F)$ and $H_{j}(B)$ are finite dimensional. We begin with some examples.

Example 5.2. Suppose the fibration (5.1) is obtained by looping a principal fibration $K(\Gamma, n) \rightarrow X \stackrel{\pi}{\rightarrow} B$ that is derived from a fibration

$$
X \stackrel{\pi}{\longrightarrow} B \stackrel{\rho}{\longrightarrow} K(\Gamma, n+1), \quad n \geq 2,
$$

where $\Gamma$ is finitely generated. We shall show that: if $H(B)$ is finite dimensional then the Serre spectral sequence for $\Omega \xi$ collapses at some $E^{r}, r<\infty$.

Indeed a classical result of Serre 32] asserts that $H^{*}(K(\pi, n+1))$ is a finitely generated algebra over the Steenrod algebra, $\mathcal{A}$. On the other hand, according to ([21]; Proposition 0.6), $\mathcal{A} . \alpha$ is finite dimensional for each $\alpha \in H^{+}(\Omega B)$. In particular, for some $k,(\mathcal{A} . \alpha)^{p^{k}}=0$. It follows that $\operatorname{Im} H^{*}(\Omega \rho)$ is finite dimensional.

Moreover, according to (30; Corollary 4.6) and [34, $\operatorname{Im} \Omega \pi=K(\Omega \rho)$. Hence $C(\Omega \pi) \cong \operatorname{Im}(\Omega \rho)$ and so $C(\Omega \pi)$ is finite dimensional. This implies collapse, by Theorem 4.10(ii). 
Example 5.3. Tensor algebras, suspensions and Ganea's spaces. When the fibre $F$ (whose loop space is the fibre of (5.1)) is a suspension a classical result of Bott and Samelson [5] asserts that $H_{*}(\Omega F)$ is a tensor algebra. Thus its Hopf centre is trivial and the Serre spectral sequence for (4.1) collapses at $E^{2}$, so that

$$
\mathbb{F}_{p} \longrightarrow H_{*}(\Omega F) \longrightarrow H_{*}(\Omega X) \longrightarrow H_{*}(\Omega B) \longrightarrow \mathbb{F}_{p}
$$

is a short exact sequence of Hopf algebras.

One example of this phenomenon is the sequence of fibrations $F_{n}(B) \stackrel{j_{n}}{\longrightarrow} E_{n}(B)$ $\stackrel{\pi_{n}}{\longrightarrow} B$ constructed by Ganea [17] in which $E_{n+1}(B)$ is the cofibre of $j_{n}$. These fibrations do not in general admit a cross-section, since by Ganea's theorem a crosssection to $\pi_{n}$ exists if and only if cat $B \leq n$. On the other hand, Ganea identifies $F_{n+1}(B)=F_{n}(B) * \Omega B$. Thus the spaces $F_{n}(B)$ are suspensions and

$$
\mathbb{F}_{p} \longrightarrow H_{*}\left(\Omega F_{n}(B)\right) \longrightarrow H_{*}\left(\Omega E_{n}(B)\right) \longrightarrow H_{*}(\Omega B) \longrightarrow \mathbb{F}_{p}
$$

is exact.

Note that other spaces (e.g. Moore spaces, the spaces $F_{n}\left\{p^{r}\right\}$, etc.) also have tensor algebras for their loop space homology, and so when $F$ is in this class the Serre spectral sequence for (5.1) also collapses at $E^{2}$.

Example 5.4. Double loop spaces. Consider a loop space fibration of the form

$$
\Omega^{2} B \longrightarrow p t \longrightarrow \Omega B
$$

with $B$ a 2-connected space. If the corresponding Serre spectral sequence collapses at some $E^{r}, r<\infty$, we deduce from Theorem 4.7 that $H_{*}\left(\Omega^{2} B\right)$ and $H^{*}(\Omega B)$ are finitely generated commutative graded algebras. This implies in turn that $H_{*}(\Omega B)$ is $p$-solvable. We remark that all this can occur, for example if $B=K(\mathbb{Z}, 3)$ or if $\Omega B$ is a finite loop space.

On the other hand, if $B$ has finite LS category and $H_{*}(B) \neq \mathbb{F}_{p}$, then this spectral sequence never collapses, since then the loop space homology, $H_{*} \Omega B$ is never $p$-solvable when $H_{*}(B) \neq \mathbb{F}_{p}$ ([8]; proof of Theorem 5.1).

The next result is analogous to a theorem of Borel [4] in the case $X=p t$.

Theorem 5.5. Suppose, $p>2$. Then the following conditions are equivalent on the fibration (5.1):

(i) $K(\Omega j)$ is a finitely generated polynomial algebra;

(ii) $C(\Omega \pi)^{\vee}$ is a finitely generated exterior algebra on elements of odd degree and $\operatorname{Im}(\Omega j)=K(\Omega \pi)$.

In this case the primitive elements in $C(\Omega \pi)$ dual to the generators of $C(\Omega \pi)^{\vee}$ will transgress to the generators of $K(\Omega j)$.

Proof. Write $K(\Omega \pi) / / \operatorname{Im}(\Omega j)=\Lambda V$, as in $\S 4$. For any $q$, if $E^{q}$ has the form $E_{*, 0}^{q} \otimes E_{0, *}^{q}$ then $d^{q}$ is determined by $d^{q}: E_{q, 0}^{q} \longrightarrow E_{0, q-1}^{q}$. The image will be the space of generators of the Hopf kernel $Z^{q}$ of $E_{0, *}^{q} \rightarrow E_{0, *}^{q}$. If $K(\Omega j)$ is a polynomial algebra then so is $Z^{q}$ (cf. Theorem 7.3) and so no elements in $U^{q+1}$ will be created (cf. $\S 6$ ). Thus $E^{q+1}$ will have the form $E_{*, 0}^{q+1} \otimes E_{0, *}^{q+1}$. It follows that if $K(\Omega j)$ is a polynomial algebra, then $V=0$. Since $p>2$ the generators of $Z^{q}$ have even degree and hence $E_{*, 0}^{q} / / E_{*, 0}^{q+1}$ is an exterior algebra on finitely many elements of odd degree. It follows that $C(\Omega \pi)^{\vee}$ is an exterior algebra on finitely many elements of odd degree whose dual primitive elements in $C(\Omega \pi)$ lift to primitive elements in $E_{*, 0}^{2}$ (Corollary 1.3) which transgress to the generators of $K(\Omega j)$. 
Conversely, suppose $C(\Omega \pi)^{\vee}$ is an exterior algebra on elements of odd degree:

$$
C(\Omega \pi)^{\vee}=\bigwedge\left(y_{1}, y_{2}, \ldots, y_{t}\right), \quad \operatorname{deg} y_{i} \text { odd } .
$$

Thus if $E^{r}=E_{*, 0}^{r} \otimes E_{0, *}^{r}$ and $d^{r} \neq 0$, then $r$ is odd and $E_{r, 0}^{r} /$ ker $d^{r}$ is spanned by elements $x_{i}$ dual to some of the $y_{i}$. It suffices to prove that the $d^{r} x_{i}$ generate a polynomial algebra. If not there would result the construction of an element $u \in U_{r, k}^{r+1}$ with $k>r$. Since all the $y_{i}$ have odd degree this $u$ could not be hit by a later differential coming from $E_{*, 0}^{s}$, and clearly $d^{s} u=0, s>r$. Thus $u$ would survive to a non-trivial element in $V$, contrary to our hypothesis.

Finally, we consider elliptic spaces. A simply connected space $T$ is $p$-elliptic [11] if the following three conditions hold:

- each $H_{i}\left(T ; \mathbb{Z}_{(p)}\right)$ is a finitely generated $\mathbb{Z}_{(p)}$-module;

- $\operatorname{cat} T_{(p)}<\infty$

- for some positive integer $r$ and some constant $C \operatorname{dim} H_{n}(\Omega F) \leq C n^{r}, n \geq 1$.

Here $T_{(p)}$ is the localization of $T$ at $p$. The space $T$ is elliptic if it is $p$-elliptic for each prime $p$ and for $p=0$. Recall further [11] that if $T$ is $p$-elliptic, then $H^{*}\left(T ; \mathbb{F}_{p}\right)$ is a finite dimensional Poincaré duality algebra.

Theorem 5.6. Suppose in the fibration $\xi: F \longrightarrow X \longrightarrow B$ that $F, X$ and $B$ are spaces of finite category whose $\mathbb{Z}_{(p)}$-homology groups are finitely generated in each degree. Then $X$ is p-elliptic if and only if $F$ and $B$ are both p-elliptic.

Proof. Since cat $F<\infty$ it follows that depth $H(\Omega F)<\infty$, the Hopf center of $H(\Omega F)$ is finitely generated, and the Serre spectral sequence for $\Omega \xi$ collapses at some $E^{r}, r<\infty$. Now Theorem A of the introduction (see the end of $\S 4$ ) asserts that $H(\Omega X)$ grows polynomially if and only if both $H(\Omega F)$ and $H(\Omega B)$ do.

\section{Part II : Differential Hopf algebras}

\section{Bigraded differential Hopf algebras}

In this section we work over a perfect field $\mathbb{K}$ of characteristic $p>0$. In defining cocommutativity, graded derivations etc. in bigraded objects $\left\{G_{i, j}\right\}$ we use the signs associated with the corresponding total gradation $G_{n}=\left\{G_{i, j}\right\}_{i+j=n}$. In particular a bigraded differential Hopf algebra is a bigraded Hopf algebra $G$ equipped with a differential $d\left(d^{2}=0\right)$ of bidegree $(-r, r-1)$, some $r$, such that $d$ is both a derivation $\left(d(x y)=(d x) y+(-1)^{\operatorname{deg} x} x d y\right)$ and a coderivation.

Our objective is to refine certain results of Browder in [6]. Thus we shall be interested in bigraded Hopf algebras $G$ satisfying the following conditions:

- $G_{0,0}=\mathbb{K} ; G=\left\{G_{i, j}\right\}_{i, j \geq 0} ;$ each $G_{i, j}$ is finite dimensional. - $G$ is cocommutative.

- The sub Hopf algebras $G_{*, 0}$ and $G_{0, *}$ are normal.

The surjections $\rho_{L}: G \longrightarrow G_{*, 0}$ and $\rho_{R}: G \longrightarrow G_{0, *}$ define the surjection

$$
\left(\rho_{L} \otimes \rho_{R}\right) \circ \Delta: G \longrightarrow G_{*, 0} \otimes G_{0, *}
$$

of bigraded Hopf algebras. Its Hopf kernel $G^{M}$ will be called the mixed sub Hopf algebra of $G$, and a natural isomorphism of bigraded Hopf algebras,

$$
\alpha_{G}: G_{*, 0} \otimes G^{M} \otimes G_{0, *} \stackrel{\cong}{\longrightarrow} G,
$$


is given by $a \otimes b \otimes c \mapsto a b c$. Indeed, $\alpha_{G}$ preserves the diagonal because multiplication in $G$ does. Moreover, because $G_{*, 0}, G^{M}$ and $G_{0, *}$ are all normal, they commute pairwise, and so $\alpha_{G}$ preserves products as well.

For example, suppose $G=\mathbb{K}[x, y, z]$ with $x, y, z$ respectively of bidegrees $(2,0)$, $(2,2),(0,2)$ with $x, z$ primitive and with $\Delta y=y \otimes 1+1 \otimes y+x \otimes z+z \otimes x$. Then $y-x z$ is primitive and in $\operatorname{ker}\left(\left(\rho_{L} \otimes \rho_{R}\right) \circ \Delta\right)$. It follows that $G^{M}=\mathbb{K}[y-x z]$.

For the rest of this section we shall fix a bigraded differential Hopf algebra $(G, d)$ in which

- $G$ is assumed to satisfy (6.1).

- $d$ has bidegree $(-r, r-1)$, some $r \geq 2$.

Lemma 6.2. The bigraded homology Hopf algebra $H(G)$ also satisfies (6.1). Thus $H(G)=H_{*, 0} \otimes H(G)^{M} \otimes H_{0, *}$.

Proof. We have only to check that $H_{0, *}$ and $H_{*, 0}$ are normal. Homology classes in these algebras are represented by cycles $x \in G_{0, *}$ and $y \in G_{*, 0}$. For any cycle $z \in G,(A d z) x$ and $(A d z) y$ are respectively cycles in $G_{0, *}$ and $G_{*, 0}$, since these are normal subalgebras. Hence $(A d[z])[x] \in H_{0, *}$ and $(A d[z])[y] \in H_{*, 0}$, as desired.

By definition the Hopf algebras $H_{*, 0}$ and $H_{0, *}$ are given by

$$
H_{*, 0}=G_{*, 0} \cap \operatorname{ker} d \quad \text { and } \quad H_{0, *}=G_{0, *} / d\left(G_{r, *}\right) .
$$

Now recall from $([29 ; \S 2)$ that the p-derived Hopf algebra of a cocommutative graded Hopf algebra, $A$, is the Hopf kernel $\langle A, A\rangle$ of the surjection $A \longrightarrow A / I$, where $I$ is the ideal generated by commutators and $p$ th powers.

Proposition 6.3. (i) The Hopf kernel of the surjection $G_{0, *} \longrightarrow H_{0, *}$ is generated by central primitive elements of $G_{0, *}$.

(ii) The sub Hopf algebra $H_{*, 0}$ contains $\left\langle G_{*, 0}, G_{*, 0}\right\rangle$. In particular it is normal in $G_{*, 0}$.

Proof. (i) The ideal $d\left(G_{r, *}\right)$ in $G_{0, *}$ is generated by $d\left(A_{r, *}\right)$, where $A=G_{*, 0} \otimes G^{M}$. Since $A$ is a subcoalgebra of $G$ and $A_{0, *}=\mathbb{K}, d x$ is primitive for $x \in A_{r, *}$. Similarly we have $[x, y]=0, y \in G_{0, *}$, so that $0=d[x, y]=[d x, y]$. Thus $d x$ is central in $G_{0, *}$.

(ii) Let $b \in\left\langle G_{*, 0}, G_{*, 0}\right\rangle$ be an element of least degree for which $d b \neq 0$. Then $d b$ is primitive of bidegree $(*, r-1)$. Since $\alpha_{G}$ is an isomorphism of coalgebras, $d b \in G^{M} \otimes G_{0, *}$. But $b$ is decomposable in $G_{*, 0}$, so that is impossible.

The next step is to consider the mixed sub Hopf algebra $H(G)^{M}$. To do so we introduce the Hopf algebras

$$
W=G_{*, 0} / / H_{*, 0} \quad \text { and } \quad Z=\operatorname{Hopf} \operatorname{ker}\left(G_{0, *} \longrightarrow H_{0, *}\right),
$$

which will play a fundamental role in the sequel. Now the sequence of Hopf algebra morphisms,

$$
\mathbb{K} \longrightarrow H_{*, 0} \stackrel{\sigma}{\rightarrow} G \stackrel{\kappa}{\longrightarrow} H_{0, *} \longrightarrow \mathbb{K}
$$

is exact at $H_{*, 0}$ and $H_{0, *}$, and the failure of exactness at $G$ is measured by the quotient Hopf algebra,

$$
(\text { Hopf } \operatorname{ker} \kappa) / / I m \sigma=W \otimes G^{M} \otimes Z .
$$


Since $H(\sigma)$ and $H(\kappa)$ are evidently respectively injective and surjective, it follows (e.g. [13]; Lemma 2.1) that

$$
H(G)^{M}=H\left(W \otimes G^{M} \otimes Z\right) .
$$

Finally in the spirit of [6] we analyze the structure of $H(G)^{M}$ under the additional hypothesis that

$$
\begin{aligned}
& G^{M} \text { is an exterior algebra, } \Lambda U, \text { on a subspace } \\
& U \text { of primitive elements of odd degree. } \\
& U_{i, j}=0 \text { unless } i<r \text { or } j<r-1 .
\end{aligned}
$$

Proposition 6.6. If $G^{M}$ satisfies (6.5) $)_{r}$ then $H(G)^{M}$ satisfies $(6.5)_{r+1}$.

Remark 6.7. In ([6]; Lemma 5.7; Theorem 5.8) Browder establishes a coalgebra isomorphism $H(G) \cong H_{0, *} \otimes \Lambda V \otimes H_{0, *}$, with $V$ consisting of primitive elements of odd degree and of bidegrees satisfying $(6.5)_{r+1}$. Combining Propositions 6.2 and 6.6 we now have this as an isomorphism of Hopf algebras. In fact, given (6.2), we could deduce (6.6) directly from Browder's result. The purpose of the independent proof we give below is to exhibit the precise interdependence between $V$ and the Hopf algebra structures in $Z$ and $W$. This interdependence will play an important role in the applications.

Proof of 6.6. Denote by $\mathbb{K}(x)$ the Hopf algebra generated by a single primitive element $x$. If in the dual Hopf algebra $\mathbb{K}(x)^{\vee}, y$ is the element dual to $x$, then we denote by $y^{(i)}$ the element dual to $x^{i}, i \geq 1$. We write $\mathbb{K}(x)=\Lambda x$ if $x^{2}=0$; in this case $x$ has odd degree unless $p=2$. Recall the classical and elementary fact that

$$
H(\Lambda x \otimes \mathbb{K}(d x))= \begin{cases}\mathbb{K} & \text { if } \mathbb{K}(d x)=\mathbb{K}[d x] \\ \mathbb{K} \oplus \mathbb{K} x(d x)^{n-1} & \text { if } \mathbb{K}(d x)=\mathbb{K}[d x] /(d x)^{n} .\end{cases}
$$

If $p=2$, then $n=2^{k}$ and $n-1$ is odd; otherwise $x$ has odd degree and $d x$ has even degree. Thus in all cases $x(d x)^{n-1}$ has odd degree.

We shall decompose $W \otimes \Lambda U \otimes Z$ as the tensor product of chain complexes of the form $\Lambda x, \Lambda x \otimes \mathbb{K}(d x)$ or $[\Lambda x \otimes \mathbb{K}(d x)]^{\vee}$, giving the graded dual ()$^{\vee}$ the obvious bigradation. It will follow that the Hilbert series $H(W \otimes \Lambda U \otimes Z)(z)$ $=\sum_{i}\left[\operatorname{dim} H_{i}(W \otimes \Lambda U \otimes Z] z^{i}\right.$ has the form

$$
H(W \otimes \Lambda U \otimes Z)(z)=\prod_{i}\left(1+z^{2 n_{i}+1}\right)
$$

This will imply that $H(W \otimes \Lambda U \otimes Z)$ has the form $\Lambda V$, where $V$ is the subspace of primitive elements, and is concentrated in odd degrees. It will follow from the construction that $V$ differs from $U$ only in bidegrees $(r, *)$ and $(*, r-1)$, whence the second assertion of the proposition.

It remains to construct the decomposition of $W \otimes \Lambda U \otimes Z$. We do this in a number of steps, using Proposition 6.3, (6.5) $r$ and the Borel structure theorem for commutative graded Hopf algebras.

(i) Since $d(U)$ consists of primitive elements of even degree,

$$
d\left(U_{i, *}\right)=0, \quad i \neq r, \quad \text { and } \quad d\left(U_{r, *}\right) \subset Z .
$$

Thus $Z$ is generated as an algebra by the primitive subspace $P=d\left(W_{r, 0}\right) \oplus d\left(U_{r, *}\right)$. By hypothesis $U_{r, j}=0$ for $j \geq r-1$; moreover $U_{r, r-2}=0$ since $U$ is concentrated 
in odd degrees. Thus $P_{i}=0$ unless $i \in[r-1,2 r-4]$. Since $Z_{+}$begins in degree $r-1$, no element of $P$ is decomposable in $Z$. It follows that

$$
Z_{+}=P \oplus Z_{+} \cdot Z_{+} .
$$

Now the Borel structure theorem (Proposition 2.1) identifies $Z$ as the tensor product of the monogenic sub Hopf algebras generated by a basis of $P$. Thus

$$
Z=\left[\bigotimes_{i} \mathbb{K}\left(d w_{i}\right)\right] \otimes\left[\bigotimes_{j} \mathbb{K}\left(d u_{j}^{\prime}\right)\right]
$$

where the $w_{i}$ are a basis of $W_{r, 0}$ and the $u_{j}^{\prime} \in U_{r, *}$ represent a basis for $U_{r, *} / U_{r, *} \cap$ ker $d$. Denote the Hopf algebra $\bigotimes_{i} \mathbb{K}\left(d w_{i}\right)$ by $B$.

(ii) Let $P^{\prime} \subset W$ be the subspace of primitive elements of degree $>r$. Then $d$ maps $P^{\prime}$ injectively into $U_{*, r-1}$. But $U_{i, r-1}=0$ for $i \geq r-1$ and so $P^{\prime}$ is concentrated in degrees $\leq 2 r-2$. On the other hand, $W_{j}=0, j<r$, and so elements in $W$ of degrees $\leq 2 r-2$ are automatically primitive; i.e.,

$$
P^{\prime}=\left\{W_{i}\right\}_{r<i \leq 2 r-2} .
$$

Put $d\left(P^{\prime}\right)=X$. We show next that

$$
d: W \rightarrow W \otimes\left(Z_{r-1} \oplus X\right) .
$$

Let $\pi: W \otimes \Lambda U \otimes Z \rightarrow W$ and $\rho: W \otimes \Lambda U \otimes Z \rightarrow \Lambda U \otimes Z$ be the obvious surjections. Clearly $\rho d(W)$ is primitive and so contained in $Z_{r-1} \oplus U_{*, r-1}$. Thus $\rho d(W)=$ $\rho d\left(W_{\leq 2 r-2}\right)=d\left(W_{\leq 2 r-2}\right)=Z_{r-1} \oplus X$. On the other hand, since $(\pi \otimes \rho) \Delta=i d$, the equation $(\pi \otimes \rho) \Delta d(w)=(\pi \otimes \rho) d \Delta w, w \in W$, leads at once to the conclusion that

$$
d: W \rightarrow W \otimes \rho d(W) .
$$

(iii) Note that $A=W \otimes \Lambda X \otimes B$ is a subdifferential Hopf algebra of $W \otimes \Lambda X \otimes$ $Z$. Let $u_{\lambda} \in U \cap \operatorname{ker} d$ represent a basis for $(U \cap \operatorname{ker} d) / X$. Then we have the decomposition

$$
W \otimes \Lambda U \otimes Z=A \otimes\left[\bigotimes_{\lambda}\left(\Lambda u_{\lambda}, 0\right)\right] \otimes\left[\bigotimes_{j}\left(\Lambda u_{j}^{\prime} \otimes \mathbb{K}\left(d u_{j}^{\prime}\right)\right)\right] .
$$

(iv) Next, we decompose $A$. Consider the commutative Hopf algebra $W^{\vee}$ dual to $W$. It is generated by elements dual to the primitive subspace of $W$ and hence (by (ii)) of degrees $\leq 2 r-2$. Since $W_{j}=0, j<r$, elements of these degrees are automatically primitive. Thus $W^{\vee}$ is the tensor product of the sub Hopf algebras generated respectively by the primitive elements of degree $r$ and the primitive elements of degree $i \in(r, 2 r-2]$. Moreover, since $W^{\vee}$ is primitively generated $W$ is commutative and its $p$ th powers vanish.

Dually, we have

$$
W=T \otimes Y,
$$

where the sub Hopf algebras $T$ and $Y$ are uniquely determined by the condition: $W_{r}$ is the primitive subspace of $T$. As in (ii) it follows that

$$
d: T \rightarrow T \otimes Z_{r-1} \quad \text { and } \quad d: Y \rightarrow Y \otimes X .
$$


In the dual Hopf algebra $\left(A^{\vee}, \delta\right)$ we have, as in (i) above, that

$$
(Y \otimes X)^{\vee}=\bigotimes_{\alpha} \mathbb{K}\left(\delta x_{\alpha}^{\vee}\right) \otimes \Lambda x_{\alpha}^{\vee}
$$

for a suitable basis $x_{\alpha}^{\vee}$ of $X^{\vee}$. (This uses the fact that $d: P^{\prime} \cong X$.) Thus

$$
A=[T \otimes B] \otimes\left[\bigotimes_{\alpha} \mathbb{K}\left(\delta x_{\alpha}^{\vee}\right) \otimes \Lambda x_{\alpha}^{\vee}\right]^{\vee}
$$

Note that if $y_{\alpha} \in Y$ is dual to $\delta x_{\alpha}^{\vee}$ then $d y_{\alpha}$ is dual to $x_{\alpha}$.

(v) Finally, we decompose $T \otimes B$; Suppose first $p=$ char $\mathbb{K}$ is odd. If $r$ is odd, then $T$ is the exterior algebra on $W_{r}$ and

$$
T \otimes B=\bigotimes_{i} \Lambda w_{i} \otimes \mathbb{K}\left(d w_{i}\right) .
$$

If $r$ is even, then $(T \otimes B)^{\vee}$ has a decomposition of this form. If char $p=2$, then squares vanish in $W$ and so $T$ is the exterior algebra on $W_{r}$ whether $r$ is even or odd. Thus $T \otimes B$ has the desired form.

The decomposition constructed this way implies that $H(W \otimes \Lambda U \otimes Z)=\Lambda V$, where $V$ is the space of primitive elements of odd degree; it is clear that $V_{i, j}=U_{i, j}$ unless $i=r$ or $j=r-1$, whence $V_{i, j}=0$ unless $i \leq r$ or $j \leq r-1$.

Corollary (of proof) 6.8. The Hopf algebras $Z$ and $W^{\vee}$ are finite tensor products of monogenic Hopf algebras, whose generators are concentrated respectively in degrees $j \in[r-1,2 r-4]$ and $i \in[r, 2 r-2]$.

Corollary (of proof) 6.9. A basis of $V$ is represented by elements of the following form:

$$
u_{\lambda}, \quad u_{j}^{\prime}\left(d u_{j}^{\prime}\right)^{p^{k_{j}}-1}, \quad y_{\alpha}^{\left(p^{l_{\alpha}}-1\right)} d y_{\alpha}, \quad w_{i}\left(d w_{i}\right)^{p^{n_{i}}-1}, \quad w_{i}^{\left(p^{m_{i}}-1\right)} d w_{i},
$$

where $y_{\alpha}, w_{i} \in P(W) ; u_{\lambda}, u_{\lambda}^{\prime}, d y_{\alpha} \in U$; and $d u_{j}^{\prime}, d w_{i} \in P(Z)$.

Corollary (of proof) 6.10. With the notation above,

(i) $V_{i, j} \cong U_{i, j}$ unless $i=r$ or $j=r-1$.

(ii) $\operatorname{dim} V_{r, *} \leq \operatorname{dim} U_{r, *}+\operatorname{dim} W_{r, 0} \leq \operatorname{dim} U_{r, *}+\operatorname{dim} G_{r, 0}$.

(iii) $\operatorname{dim} V_{*, r-1} \leq \operatorname{dim} U_{*, r-1}+\operatorname{dim} \bar{Z}_{0, r-1} \leq \operatorname{dim} U_{*, r-1}+\operatorname{dim} G_{0, r-1}$.

(iv) $\operatorname{dim} V_{r, j} \leq \operatorname{dim} U_{r, j}, j \leq r-2$.

(v) $\operatorname{dim} V_{i, r-1} \leq \operatorname{dim} U_{i, r-1}, i \leq r-1$.

In particular, if $U$ is finite dimensional, so is $V$.

\section{Spectral Sequences of Hopf Algebras}

In this section we consider a spectral sequence

$$
\left(E_{*, *}^{r}, d^{r}\right), \quad r \geq 2
$$

of bigraded differential Hopf algebras, satisfying the following conditions:

- $E_{0,0}^{2}=\mathbb{K} ; E^{2}=\left\{E_{i, j}^{2}\right\}, \quad i, j \geq 0 ; \quad$ each $E_{i, j}^{2}$ is finite dimensional.

- $E^{2}$ is cocommutative.

- $E^{2}=E_{*, 0}^{2} \otimes E_{*, 0}^{2}$ as bigraded Hopf algebras.

- $d^{r}$ has bidegree $(-r, r-1)$. 
During the entire section the spectral sequence will be fixed and assumed to satisfy (7.1). As usual the bigraded Hopf algebra $E_{*, *}^{\infty}$ associated with the spectral sequence is defined by

$$
E_{i, j}^{\infty}=E_{i, j}^{r}, \quad r>\max (i, j+1) .
$$

It is immediate from Lemma 6.2 and Proposition 6.6 that each term $\left(E^{r}, d^{r}\right)$ satisfies both conditions $(6.1)$ and $(6.5)_{r}$ of the preceding section. Thus we have the following refinement of ([6]; Theorem 5.8).

Theorem 7.2. The spectral sequence $\left(E^{r}, d^{r}\right)$ above satisfies:

(i) For $r \leq \infty, E_{*, *}^{r}=E_{*, 0}^{r} \otimes \Lambda U^{r} \otimes E_{0, *}^{r}$ as bigraded Hopf algebras, where $U^{r}$ consists of primitives of odd degrees.

(ii) In the sequence $E_{*, 0}^{2} \supset \cdots \supset E_{*, 0}^{r} \supset \cdots$, each $E_{*, 0}^{r+1}$ is normal in $E_{*, 0}^{r}$. The Hopf algebra $A_{r}$ dual to $E_{*, 0}^{r} / / E_{*, 0}^{r+1}$ is generated by finitely many primitive elements, whose degrees $i$ satisfy: $i=r$, or else $i$ is an integer in $[r+1,2 r-2]$ of the form $i=2 n$ if $p=2$ and $2 p n+2$ if $p>2$.

(iii) In the sequence $E_{0, *}^{2} \rightarrow \cdots \rightarrow E_{0, *}^{r} \rightarrow \cdots$, the Hopf kernel $Z^{r}$ of the surjection $E_{0, *}^{r} \longrightarrow E_{0, *}^{r+1}$ is central and generated by finitely many primitive elements, whose degrees $j$ satisfy: $j=r-1$, or else $j$ is an integer in $[r, 2 r-4]$ of the form $j=2 n$ if $p=2$ and $2 p n-2$ if $p>2$.

(iv) For $r \leq \infty$ the bidegrees $(i, j)$ of the elements of $U_{*, *}^{r}$ have one of the following two forms:

$$
(i, j)=\left\{\begin{array}{l}
(p m-q, q-1), \quad \text { some } q \in(1, r) \\
\text { or }(q+1, p m-q), \quad \text { some } q \in(0, r-1),
\end{array}\right.
$$

with $q \leq m<2 q-1$. In the first case $m$ is the degree of a generator of $A_{q}$. Thus either $m=q$ or $U_{m-q, q-1}^{q} \neq 0$. In the second case, $m$ is the degree of a generator of $Z^{q}$. Thus $m=q$ or $U_{q+1, m-q}^{q} \neq 0$.

(v) In particular, for $r<\infty, U^{r}$ is finite dimensional and concentrated in the union of the columns $i<r$ and the rows $j<r-1$. The space $U^{\infty}$ may be infinite dimensional, but will still have finite dimensional rows and columns.

Proof. The assertions for $r<\infty$ follow directly by induction from Proposition 6.6 and Corollary 6.9. The assertions for $r=\infty$ follow by a limit argument.

In the spectral sequence (7.1) consider the short exact sequence of Hopf algebras

$$
\mathbb{K} \longrightarrow K \longrightarrow E_{0, *}^{2} \stackrel{\eta}{\rightarrow} E_{0, *}^{\infty} \longrightarrow \mathbb{K},
$$

where $K$ is the Hopf kernel of the edge homomorphism $\eta$. It is the union of the Hopf kernels $K^{r}$ of $\eta^{r}: E_{0, *}^{2} \longrightarrow E_{0, *}^{r}, r \geq 2$, and this identifies $Z^{r}=K^{r+1} / / K^{r}$.

Theorem 7.3. If $K$ is commutative, then there are graded algebra splittings for the surjections $K^{r+1} \rightarrow Z^{r}$, which then define an isomorphism of graded algebras, $K^{r+1} \cong K^{r} \otimes Z^{r}$. In particular

$$
K \cong \bigotimes_{r \geq 2} Z^{r}
$$

On the other hand, if $E_{*, 0}^{\infty}$ is normal in $E_{*, 0}^{2}$ we have short exact sequences of Hopf algebras

$$
\mathbb{K} \longrightarrow E_{*, 0}^{r+1} / / E_{*, 0}^{\infty} \longrightarrow E_{*, 0}^{r} / / E_{*, 0}^{\infty} \longrightarrow E_{*, 0}^{r} / / E_{*, 0}^{r+1} \longrightarrow \mathbb{K}
$$


Theorem 7.4. If $E_{*, 0}^{\infty}$ is normal in $E_{*, 0}^{2}$, then there are graded coalgebra splittings for the inclusions $E_{*, 0}^{r+1} / / E_{*, 0}^{\infty} \longrightarrow E_{*, 0}^{r} / / E_{*, 0}^{\infty}$, which then define isomorphisms of graded coalgebras, $E_{*, 0}^{r} / / E_{*, 0}^{\infty} \cong E_{*, 0}^{r+1} / / E_{*, 0}^{\infty} \otimes E_{*, 0}^{r} / / E_{*, 0}^{r+1}$. In particular,

$$
E_{*, 0}^{2} / / E_{*, 0}^{\infty} \cong \bigotimes_{r \geq 2} E_{*, 0}^{r} / / E_{*, 0}^{r+1} .
$$

Proof of Theorem 7.3. The degree of a generator of $K^{r}$ is the degree of a generator of some $Z^{q}, q<r$. Hence by Theorem 7.2(iii), $K^{r}$ is generated in degrees $\leq 2(r-3)$. On the other hand, again from Theorem 7.2(iii), $Z^{r}$ is generated in degrees $\geq r-1$. Thus

$$
Q_{n}\left(Z^{r}\right) \neq 0 \Longrightarrow n \geq r-1 \Longrightarrow n p^{k}>2(r-3) \Longrightarrow Q_{n p^{k}}\left(K^{r}\right)=0, \quad k \geq 1 .
$$

Now apply Proposition 2.1.

Proof of Theorem 7.4. Consider the exact sequence of commutative graded Hopf algebras,

$$
\mathbb{K} \longrightarrow A_{r} \longrightarrow\left(E_{*, 0}^{r} / / E_{*, 0}^{\infty}\right)^{\vee} \longrightarrow\left(E_{*, 0}^{r+1} / E_{*, 0}^{\infty}\right)^{\vee} \longrightarrow \mathbb{K}
$$

dual to the exact sequence above by the very definitions, $E_{*, 0}^{r+1} / E_{*, 0}^{\infty}$ vanishes in degrees $\leq r$. On the other hand, according to Theorem 7.2(ii), $A_{r}$ is generated in degrees $\leq 2(r-1)$. Apply Proposition 2.1.

Proposition 7.5. In the spectral sequence of Theorem 7.2 the following conditions are equivalent:

(i) $E^{\infty}=E^{r}$, some $r<\infty$.

(ii) The Hopf kernel $K$ of the edge homomorphism $E_{0, *}^{2} \longrightarrow E_{0, *}^{\infty}$ is finitely generated.

(iii) For some $r<\infty, E_{0, *}^{r} \stackrel{\cong}{\longrightarrow} E_{0, *}^{\infty}$.

(iv) The algebra dual to the Hopf cokernel $C$ of the edge homomorphism $E_{*, 0}^{\infty} \longrightarrow$ $E_{0, *}^{2}$ is finitely generated.

(v) For some $r<\infty, E_{*, 0}^{\infty} \stackrel{\cong}{\longrightarrow} E_{*, 0}^{r}$.

Proof. Clearly (i) $\Longrightarrow($ iii $) \Longrightarrow$ (ii), since the Hopf kernel of $E_{0, *}^{i+1} \longrightarrow E_{0, *}^{i}$ is finitely generated by Theorem 7.2 (ii). To show (ii) $\Longrightarrow$ (iii), note that since $K$ is the increasing union of the Hopf kernels $K^{r}$ of the morphisms $E_{0, *}^{2} \longrightarrow E_{0, *}^{r}$, it follows that $K=K^{r}$, some $r$. This implies that the surjections $E_{0, *}^{i} \longrightarrow E_{0, *}^{i+1}$ are injective for $i \geq r$ and so $E_{0, *}^{r} \stackrel{\cong}{\cong} E_{0, *}^{\infty}$.

To show (iii) $\Longrightarrow(\mathrm{i})$ note that $d^{i}: U^{i} \oplus E_{i, 0}^{i} \longrightarrow E_{0, *}^{i}$. Hence for $i \geq r, d^{i}$ vanishes in $U^{i}$ and in $E_{i, 0}^{i}$. It follows that we have a linear map $U^{i} \longrightarrow U^{i+1}$. Moreover from the explicit description of the creation of $U^{i+1}$ given in Corollary 6.9 and the fact that $d^{i}$ vanishes in $U^{i}$ and $E_{i, 0}^{i}$ we deduce that for $i \geq r$

$$
U_{j, \ell}^{i} \longrightarrow U_{j, \ell}^{i+1} \text { is surjective, } j<\ell \text {. }
$$

Since $U^{r}$ is finite dimensional (Theorem $7.2(\mathrm{v})$ ) we conclude that for some $q \in[r, \infty$ )

$$
U_{j, \ell}^{i} \stackrel{\cong}{\longrightarrow} U_{j, \ell}^{i+1}, \quad j<\ell, \quad i \geq q .
$$

Hence $d^{i}\left(E_{*, 0}^{i}\right) \cap\left(U^{i} \oplus E_{0, *}^{i}\right)=0$. Now step (ii) in the proof of Proposition 6.6 shows that $d^{i}=0$ in $E_{*, 0}^{i}$ for $i \geq q$. Hence $d^{i}=0, i \geq q$, i.e. the spectral sequence collapses at $E^{q}$. 
The equivalences (i) $\Longleftrightarrow$ (iv) $\Longleftrightarrow(\mathrm{v})$ follow from an identical argument using the dual spectral sequence.

\section{REFERENCES}

[1] D.J. Anick, Hopf algebras up to homotopy, J. of Amer. Math. Soc., 2 (1989), 417-453. MR 90c:16007

[2] S. Araki, Steenrod reduced powers in the spectral sequence associated with a fibering, I, II, Mem. Fac. Sci. Kyushu Univ. Ser. A. Math. II (1957), 15-64, 81-97. MR 21:4419

[3] A. Borel, Sur la cohomologie des espaces fibrés principaux et des espaces homogènes de groupes de Lie compacts, Ann. Math., 57 (1953), 115-207. MR 14:490e

[4] A. Borel, Topics in the homology theory of fibre bundles, Lectures Notes in Math., 36 (1967), 1-93. MR 36:4559

[5] R. Bott, H. Samelson, On the Pontrjagin product in spaces of paths, Comm. Math. Helv., 27 (1953), 320-337. MR 15:643b

[6] W. Browder, On differential Hopf algebras, Trans. Amer. Math. Soc., 107 (1963), 153-176. MR 26:3061

[7] Y. Félix and S. Halperin, Rational $L S$ category and its applications, Trans. Amer. Math. Soc., 273 (1982), 1-37. MR 84h:55011

[8] Y. Félix, S. Halperin, J.M. Lemaire and J.-C. Thomas, Mod $p$ loop space homology, Invent. Math., 95 (1989), 247-262. MR 89k:55010

[9] Y. Félix, S. Halperin, and J.-C. Thomas, Hopf algebras of polynomial growth, Journal of Algebra, 125 (1989), 408-417. MR 90j:16021

[10] Y. Félix, S. Halperin and J.-C. Thomas, Elliptic Hopf algebras, J. London Math. Soc., 43 (1991), 545-555. MR 92i:57033

[11] Y. Félix, S. Halperin and J.-C. Thomas, Elliptic spaces, Bull. Amer. Math. Soc., 25 (1991), 69-73. MR 92m:55009

[12] Y. Félix, S. Halperin and J.-C. Thomas, Adams' Cobar equivalence, Trans. Amer. Math. Soc., 329 (1992), 531-549. MR 92e:55007

[13] Y. Félix, S. Halperin and J.-C. Thomas, Torsion in loop space homology, J. Reine Angew. Math., 432 (1992), 77-92. MR 93i:55012

[14] Y. Félix, S. Halperin and J.-C. Thomas, The category of a map and the grade of a module, Israel J. of Math., 78 (1992), 177-196. MR 94e:55021

[15] Y. Félix, S. Halperin and J.-C. Thomas, Elliptic spaces II, Enseignement Mathématique, 39 (1993), 25-32. MR 94f:55008

[16] Y. Félix and J.-C. Thomas, Module d'holonomie d'une fibration, Bull. Soc. Math. France, 113 (1985), 255-258. MR 87i:55022

[17] T. Ganea, A generalization of the homology and the homotopy suspension, Comm. Math. Helv., 39 (1965), 295-322. MR 31:4033

[18] P. Goerss, J. Lannes and F. Morel, Vecteurs de Witt non commutatifs et représentabilité de l'homologie modulo $p$, Invent. Math. 108 (1992), 163-227. MR 93e:55014

[19] S. Halperin, Rational fibrations, minimal models and fiberings of homogeneous spaces, Trans. Amer. Math. Soc., 244 (1978), 199-224. MR 58:24264

[20] S. Halperin, Universal enveloping algebras and loop space homology, J. Pure and Applied Algebra, 83 (1992), 237-282. MR 93k:55014

[21] J. Lannes and L. Schwartz, A propos des conjectures de Serre et Sullivan, Invent. Math., 83 (1986), 593-603. MR 87f:55009

[22] J.P. Lin, Torsion in $H$-spaces, I, Annals of Math., 103 (1976), 457-487. MR 54:13911

[23] J. McCleary, User's guide to spectral sequences, (1985), Publish or Perish, Inc. Math. Lect., Series 12. MR 87f:55014

[24] J. McCleary, Homotopy theory and closed geodesics, in Homotopy theory and related topics, Proceedings, Kinosaki, 1988, M. Mimura editor, Lecture Notes in Mathematics 1418 (1990) Springer-Verlag, 86-94. MR 91e:57060

[25] C.A. McGibbon and C.W. Wilkerson, Loop spaces of finite complexes at large primes, Proc. of the Amer. Math. Soc., 96 (1986), 698-702. MR 87h:55015

[26] J. Milnor, Construction of universal bundles, I, Annals of Math., 63 (1958), 272-284. MR $17: 994 \mathrm{~b}$ 
[27] J. Milnor and J.C. Moore, The structure of Hopf algebras, Annals of Math., 81 (1965), 211-264. MR 30:4259

[28] M. Mimura and H. Toda, Topology of Lie groups, I and II, Translations of Mathematical Monographs, 91, American Mathematical Society, 1991. MR 92h:55001.

[29] J.C. Moore and L. Smith, Hopf algebras and multiplicative fibrations, I, Amer. J. Math., 90 (1968), 752-780. MR 38:2772

[30] J.C. Moore and L. Smith, Hopf algebras and multiplicative fibrations, II, Amer. J. Math., 90 (1968), 1113-1150. MR 38:6599

[31] J.P. Serre, Homologie singulière des espaces fibrés. Applications, Annals of Math., 54 (1951), 425-505. MR 13:574g

[32] J.P. Serre, Cohomologie modulo 2 des complexes d'Eilenberg-MacLane, Comm. Math. Helv., 27 (1953), 198-232. MR 15:643c

[33] W.M. Singer, Extension theory for connected Hopf algebras, J. Algebra, 21 (1972), 1-16. MR 47:8597

[34] L. Smith, The cohomology of stable two stage Posnikov systems, Illinois J. of Math., 11 (1967), 310-329. MR 34:8406

[35] R. Vázquez Garcia, Note on Steenrod squares in the spectral sequence of a fibre space, Bol. Soc. Math Mexicana, 2 (1957), 1-8. MR 19:973d

[36] G.W. Whitehead, Elements of homotopy theory, Graduate Texts in Mathematics, 61, Springer-Verlag, 1978. MR 80b:55001

Institut de Mathématiques, Université de Louvain-la-Neuve, B-1348 Louvain-LANeuve, Belgium

College of Computer, Mathematical and Physical Sciences, University of Maryland, College Park, Maryland 20742-3281

Faculté des Sciences, Université d’Angers, 49045 Bd Lavoisier, Angers, France 